\title{
4 Der Kalte Krieg erreicht Südasien
}

Die eskalierende Konfrontation in und um Ostpakistan fiel zeitlich nicht nur mit einem bedeutenden Wandel in den deutsch-deutschen Beziehungen zusammen, sondern traf darüber hinaus auch auf eine sich völlig verändernde Machtkonstellation im gegeneinander gerichteten Ringen der beiden großen Systemblöcke in Ost und West. Die unerwartete Annäherung zwischen den USA und der VR China im Juli 1971 stieß die politischen Führungen in Moskau und Neu-Delhi gleichermaßen vor den Kopf. ${ }^{1}$ Die indische Regierung unter Indira Gandhi sah sich nun aus mehreren Richtungen gleichzeitig von den USA bedrängt: Einerseits half die Nixon-Administration der pakistanischen Zentralregierung bei der Unterdrückung der von Indien unterstützten ostbengalischen Unabhängigkeitsbewegung und andererseits kooperierte sie nun auch noch mit der Indien ebenfalls unfreundlich gesinnten VR China. Dies veranlasste die indische Regierung, Anfang August 1971 ihrerseits einen Freundschaftsvertrag mit der UdSSR abzuschließen. Erst diese Konstellation machte den regionalen Konflikt in Südasien zu einer Krise des Kalten Krieges. ${ }^{2}$ Auch auf die Südasienpolitik der beiden deutschen Staaten sollte dieses changierende weltpolitische Umfeld einen wichtigen Einfluss haben.

\subsection{Ein heißer Krieg im Kalten Krieg}

\author{
Mr. President, for an elected dictator you ran a lousy election. \\ Henry Kissinger zu General Yahya Khan ${ }^{3}$
}

Bereits seit 1954 verband Pakistan und die USA eine strategische Partnerschaft, ${ }^{4}$ die fünf Jahre später durch den Abschluss eines Kooperationsvertrages (einschließlich einer Beistandsklausel im Verteidigungsfall) noch einmal unterstrichen wurde. Seither war die pakistanische Armee auf Rüstungshilfe aus den USA unbedingt angewiesen - eine Tatsache, der sich die verschiedenen Regie-

1 Macmillan: Nixon and Mao, S. xx.

2 Wagner: Die „verhinderte“ Großmacht? S. 106.

3 Zit. n. Macmillan: Nixon and Mao, S. 220.

4 McMahon: On the Periphery of a Global Conflict: India and the Cold War, 1947-1991, S. 282; Smith: New Bottles for New Wine, S. 580-1. Hintergrund war, dass die USA nach dem Koreakrieg eine mögliche sowjetische Expansion in Asien und vor allem eine Bedrohung des Nahen Ostens fürchteten. S. Raghavan: 1971. A Global History of the Creation of Bangladesh, S. 3 bzw. McMahon: Cold War on the Periphery. The United States, India and Pakistan, S. 338.

O Open Access. (C) 2020 Alexander Benatar, publiziert von De Gruyter. () BY-Nc-ND Dieses Werk ist lizenziert unter der Creative Commons Attribution-NonCommercial-NoDerivatives 4.0 Lizenz. https://doi.org/10.1515/9783110682038-004 
rungen in Washington ebenso bewusst waren wie der, dass jede Waffenlieferung an Pakistan dessen Nachbarn Indien ein Dorn im Auge sein musste. ${ }^{5}$ Mit ihrem Bekenntnis zu Pakistan nahmen die USA eine mögliche Verschlechterung ihrer bilateralen Beziehungen zu Indien also bewusst in Kauf.

Bei Amtsantritt von Präsident Richard Nixon im Januar 1969 war das wichtigste Ziel der neuen US-Administration in Asien der gesichtswahrende Abzug ihrer Truppen aus Vietnam. Um dieses Ziel zu erreichen, war Nixon bereit, mit einem wesentlichen außenpolitischen Prinzip seiner Vorgänger $\mathrm{zu}$ brechen: der bis dahin als offizielle Vertretung Chinas anerkannten Republik China auf Taiwan unter Führung von Chiang Kai-sheks antikommunistisch eingestellter Kuomintang in den Rücken zu fallen und statt ihrer die kommunistische VR China anzuerkennen. Letztere versorgte Nordvietnam mit Ausrüstung und Truppen für den Kampf gegen die USA. Nixon hoffte, dass eine freundlich gesinnte VR China Druck auf ihre vietnamesischen Verbündeten ausüben würde, mit den USA endlich in Verhandlungen über ein Ende des verlustreichen Krieges in Südostasien zu treten. ${ }^{6}$

Bald nach seiner Amtseinführung begaben sich Präsident Nixon und sein Nationaler Sicherheitsberater Henry A. Kissinger auf die Suche nach einem zuverlässigen und diskreten Partner, der den Kontakt für geheime Verhandlungen mit der Pekinger Führung herstellen könnte. Recht schnell fiel die Wahl auf Pakistan - den einzigen freundlich gesinnten Nachbarn der VR China, der auch für die USA als Ansprechpartner akzeptabel war. ${ }^{7}$ Bereits im Sommer 1969 bat Präsident Nixon den neuen pakistanischen Präsidenten General Yahya Khan, die amerikanisch-chinesische Annäherung zu vermitteln. ${ }^{8}$ Dieser erklärte sich hierzu gern bereit und die anschließende Geheimkorrespondenz zwischen dem Weißen Haus und Peking erfolgte fortan über Islamabad. ${ }^{9}$

Im Gegenzug für seine Hilfe forderte Yahya Khan von Nixon allerdings auch gewisse Gegenleistungen - vor allem in Form von Waffenlieferungen. Zum größten Verdruss der indischen Regierung versprach die US-Regierung ihm im Oktober 1970 tatsächlich die Lieferung von Kampfflugzeugen und 300 Schützen-

5 Matinuddin: Tragedy of Errors, S. 311; Heß: Bangladesch. Tragödie einer Staatsgründung, S. 109; Raghavan: 1971. A Global History of the Creation of Bangladesh, S. 83.

6 Ebd., S. 85; 191; vgl. Guha: India after Gandhi, S. 459; Macmillan: Nixon and Mao, S. xxi; Westad: The Cold War, S. 406. Ziel Nixons war es außerdem, mit einer Annäherung an die VR China auch die UdSSR unter Druck setzen und dadurch die laufenden Strategic Arms Limitation Talks (SALT) beschleunigen zu können. S. Kux: The United States and Pakistan, S. 182.

7 Macmillan: Nixon and Mao, S. xx.

8 Kux: The United States and Pakistan, S. 182; Macmillan: Nixon and Mao, S. 164.

9 Macmillan: Nixon and Mao, S. 173. 
panzern. ${ }^{10}$ „Nobody has occupied the White House who is friendlier to Pakistan than me“, ${ }^{11}$ versicherte Nixon daraufhin dem pakistanischen Präsidenten und bat ihn, Peking seinen Gesprächswunsch zu übermitteln. Im November 1970 kam Yahya Khan dieser Bitte nach. ${ }^{12}$

In Peking hatten hochrangige Militärs bereits ein Jahr zuvor eine Annäherung an die USA befürwortet, sodass der Premierminister der VR China, Zhou Enlai, dem Weißen Haus über den pakistanischen Botschafter in den USA bald positive Antwort ausrichten ließ. Auch von chinesischer Seite wurde damit die Vermittlerrolle Pakistans akzeptiert. Der Gesprächskanal zwischen Peking und Washington war nun eröffnet, ein Treffen auf Ebene der politischen Führung stand indes noch aus. Auch hierfür hofften Nixon und Kissinger unbedingt auf Yahya Khans diskrete Hilfe, denn von den geheimen Vorbereitungen für dieses Treffen sollte selbst das US-amerikanische State Department erst so spät wie möglich erfahren. ${ }^{13}$

Die sich im Frühjahr 1971 andeutende Krise in Ostpakistan versuchten der amerikanische Präsident und sein Sicherheitsberater so lange wie möglich zu ignorieren, um Yahya Khan den Rücken frei zu halten. ${ }^{14}$ Zwar drängten sie den pakistanischen Präsidenten dazu, als Zeichen guten Willens die Hilfsangebote des UN-Generalsekretärs U Thant anzunehmen, ${ }^{15}$ in erster Linie waren die beiden in Südasien aber darauf bedacht, „to avoid adding another complication to our agenda”, wie Kissinger es später formulierte. ${ }^{16}$

Der Aufforderung des US-amerikanischen Generalkonsuls in Dhaka, Archer K. Blood, die US-Regierung möge das blutige Vorgehen der pakistanischen Armee gegen die eigene Bevölkerung in Ostbengalen offiziell verurteilen, kam die NixonAdministration Ende März 1971 nicht nach. In Indien beschuldigte man die USRegierung daraufhin, in Ostpakistan zu einem Völkermord beizutragen. Tatsäch-

10 Kux: The United States and Pakistan, S. 183; Raghavan: 1971. A Global History of the Creation of Bangladesh, S. 84-6.

11 Zit. n. Kux: The United States and Pakistan, S. 214; McMahon: On the Periphery of a Global Conflict: India and the Cold War, 1947-1991, S. 294.

12 Matinuddin: Tragedy of Errors, S. 305; Kux: The United States and Pakistan, S. 183; Raghavan: 1971. A Global History of the Creation of Bangladesh, S. 87.

13 Ebd., S. 192-3; Kux: The United States and Pakistan, S. 184-5.

14 Vor diesem Hintergrund ist auch Kissingers abschlägige Antwort auf Indira Gandhis Aufforderung zu verstehen, die USA sollten ihre Hilfslieferungen an das Land einstellen, s. Shirin Tahir-Kheli: India, Pakistan, and the United States. Breaking with the Past. New York: Council on Foreign Relations Press 1997, S. 37.

15 Raghavan: 1971. A Global History of the Creation of Bangladesh, S. 147-8.

16 Zit. n. Kux: The United States and Pakistan, S. 179. 
lich setzte die pakistanische Armee dort umfangreich amerikanische Waffen ein. ${ }^{17}$ Berichte auch der eigenen Diplomaten vor Ort über Massaker und massive Menschenrechtsverletzungen (am bekanntesten das sog. „Blood-Telegram“ ${ }^{18}$ ) wurden aufgrund des höherwertigen Interesses Nixons an einer pakistanisch vermittelten Annäherung an die VR China bewusst ignoriert. ${ }^{19}$

Während das Sterben in Ostbengalen weiterging, erhielt Kissinger am 27. April 1971 über den pakistanischen Botschafter in Washington die erhoffte Antwort aus der VR China: Zhou Enlai war mit einem Treffen einverstanden und arrangieren sollte es der bereits bewährte Yahya Khan. ${ }^{20}$ Anfang Mai 1971 beschloss Präsident Nixon, diesem in Ostbengalen freie Hand zu lassen. Als übergeordnetes Ziel sah auch er zwar grundsätzlich eine größere Autonomie für Ostpakistan, Yahya Khan sollte auf dem Weg dorthin aber keinesfalls unter Druck gesetzt werden. ${ }^{21}$

Bis Ende Mai 1971 hätte für die Nixon-Administration vielleicht noch die Möglichkeit bestanden, durch Einfrieren wichtiger Entwicklungshilfezahlungen an die pakistanische Zentralregierung Druck auf Yahya Khan zugunsten einer politischen Lösung auszuüben. Innenpolitisch wäre eine solche Haltung für Nixon vermutlich sogar der Weg des geringeren Widerstands gewesen, da der US-Kongress seine Zurückhaltung im Konflikt um Ostpakistan zu dieser Zeit zunehmend scharf kritisierte. ${ }^{22}$ Auch hier verdammte ihr überwiegendes Interesse an einer Aussöhnung mit der VR China und damit an einem raschen Ende des Krieges in

17 Ebd., S. 187; William J. Barnds: India, Pakistan and the Great Powers. New York / Washington / London: Praeger Publishers 1972, S. 245-6; William P. Rogers: United States Foreign Policy 1971. A Report of the Secretary of State. Washington, D.C.: Department of State 1972, S. 115; Gerd Linde: Bangla Desh. Indien und die Großmächte im Pakistanischen Konflikt. Stuttgart: Kohlhammer 1972, S. 111; Raghavan: 1971. A Global History of the Creation of Bangladesh, S. 84.

18 „Our government has failed to denounce the suppression of democracy. Our government has failed to denounce atrocities. Our government has failed to take forceful measures to protect its citizens while at the same time bending over backwards to placate the West Pak[istan] dominated government and to lessen any deservedly negative international public relations impact against them. Our government has evidenced what many will consider moral bankruptcy (...) But we have chosen not to intervene, even morally, on the grounds that the Awami conflict, in which unfortunately the overworked term genocide is applicable, is purely an internal matter of a sovereign state. Private Americans have expressed disgust. We, as professional civil servants, express our dissent with current policy and fervently hope that our true and lasting interests here can be defined and our policies redirected." U.S. Consulate (Dacca) Cable, Dissent from U.S. Policy Toward East Pakistan, April 6, 1971, zit. n. Raghavan: 1971. A Global History of the Creation of Bangladesh, S. 89.

19 Ebd., S. 89-90.

20 Kux: The United States and Pakistan, S. 188.

21 Raghavan: 1971. A Global History of the Creation of Bangladesh, S. 81; 92.

22 Ebd., S. 94; 100; Sargent: A Superpower Transformed, S. 87. 
Südostasien Nixon und Kissinger zur Tatenlosigkeit. Der Bangladeschkrieg ereignete sich insofern also auch im Windschatten des Vietnamkriegs.

Unter heftigem Protest der indischen Regierung lieferten die USA noch im Frühsommer 1971 weitere Rüstungsgüter nach Pakistan. ${ }^{23}$ Als Henry Kissinger Anfang Juli 1971 Neu-Delhi besuchte und Indira Gandhi ihn um US-amerikanische Vermittlung zwischen West- und Ostpakistan bat, ging er hierauf nicht ein, sondern versuchte stattdessen vergeblich, die indische Premierministerin $\mathrm{zu}$ überzeugen, ihre Unterstützung für die Mukti Bahini zu beenden. Nach seinem Gespräch mit Indira Gandhi hielt Kissinger einen baldigen Kriegsausbruch für ausgesprochen wahrscheinlich. ${ }^{24}$ Von Indien aus flog er weiter nach Pakistan. Die amerikanische Unterstützung Yahya Khans sollte sich nun auszahlen: Am 9. Juli 1971 reiste Kissinger mit pakistanischer Hilfe heimlich nach Peking, um dort einen Besuch Präsident Nixons im nächsten Jahr zu vereinbaren. ${ }^{25}$

Nachdem der pakistanische Präsident sich als loyal erwiesen hatte, glaubten Nixon und Kissinger, ihren neu gewonnen Partnern in Peking ihre Zuverlässigkeit beweisen zu müssen, indem sie sich mit dem gemeinsamen Freund Pakistan weiterhin solidarisch zeigten. ${ }^{26}$ Über dieses Ziel schossen sie allerdings hinaus: Zwar drängte Zhou Enlai wie Henry Kissinger auf eine indische Mäßigung in Ostpakistan, Kissinger überschätzte jedoch deutlich die chinesische Bereitschaft, tatsächlich zugunsten Pakistans in einen militärischen Konflikt

23 Raghavan: 1971. A Global History of the Creation of Bangladesh, S. 102. Am 6. April verhängte das US-Außenministerium zwar ein zum 25. März 1971 rückwirkendes Embargo auf Rüstungslieferungen nach Pakistan, die Auslieferung bereits vor diesem Zeitpunkt genehmigter Güter endete jedoch erst mit dem Totalembargo von Dezember 1971, nachdem im Juli auf öffentlichen Druck selbst die finanziell weit bedeutendere Ernährungshilfe für Pakistan ausgesetzt, bzw. gezielt nach Ostpakistan umgelenkt worden war. S. Sisson / Rose: War and Secession, S. 256-7; Linde: Bangla Desh. Indien und die Großmächte im Pakistanischen Konflikt, S. 112. Zuvor ging der Löwenanteil der von den USA geleisteten Entwicklungshilfe an den pakistanischen Westflügel, s. Van Schendel: A History of Bangladesh, S. 145.

24 Kux: The United States and Pakistan, S. 191.

25 Guha: India after Gandhi, S. 454; s. auch Wagner: Die „verhinderte“ Großmacht? S. 184-5. Zum Hintergrund dieses konspirativen Treffens in Peking, einschließlich des Einsatzes eines Kissinger-Doubles, der von Islamabad aus zum Schein in die pakistanischen Berge fuhr, s. Matinuddin: Tragedy of Errors, S. 306 und Xia / Tudda, Beijing, 1972, S. 51-53. Auch das eigene Außenministerium sollte von diesem Coup Nixons und Kissingers nichts erfahren, s. Raghavan: 1971. A Global History of the Creation of Bangladesh, S. 85. Selbst die US-amerikanischen Geheimdienste wurden über Kissingers eigentliches Reiseziel im Dunkeln gelassen. S. Macmillan: Nixon and Mao, S. 191.

26 Raghavan: 1971. A Global History of the Creation of Bangladesh, S. 106; Macmillan: Nixon and Mao, S. 222. 
mit Indien einzutreten. ${ }^{27}$ Bereits als Zhou Enlai dem pakistanischen Präsidenten Yahya Khan im April 1971 die Solidarität der VR China im Falle eines indischen Angriffs versicherte, hatte er unmissverständlich klar gemacht, dass die pakistanische Regierung keinesfalls auf ihre militärische Unterstützung zählen durfte. ${ }^{28}$ Im Übrigen wäre ein chinesisches Eingreifen in diesen Konflikt auch deshalb nur schwer zu rechtfertigen gewesen, da die pakistanische Armee in Ostpakistan unter anderem pro-chinesische Kräfte unterdrückte. ${ }^{29}$ Peking übte sich also in Zurückhaltung.

Dennoch teilte Kissinger der indischen Regierung nach seiner Chinareise mit, dass die USA Indien in einem erneuten indisch-pakistanischen Krieg niemals gegen eine chinesische Intervention zugunsten Pakistans unterstützen würden. ${ }^{30}$ Aus indischer Sicht war dies der Tropfen, der das Fass zum Überlaufen brachte. Indira Gandhi beschloss nun, dem langjährigen sowjetischen Drängen auf Unterzeichnung eines bilateralen Freundschaftsvertrages nachzugeben. ${ }^{31}$

\section{Indische Bündnisfreiheit am Scheideweg}

Über Jahrzehnte erstritten, war es seit Erlangung der Unabhängigkeit im Jahre 1947 wichtigstes außenpolitisches Anliegen Indiens, sich diese Unabhängigkeit auch im Blocksystem des Kalten Krieges zu bewahren. ${ }^{32}$ Bereits im September

27 Kux: The United States and Pakistan, S. 192; Raghavan: 1971. A Global History of the Creation of Bangladesh, S. 197-8

28 Barnds: India, Pakistan and the Great Powers, S. 244; Wagner: Die „verhinderte“ Großmacht? S. 186; Matinuddin: Tragedy of Errors, S. 319; so auch Raghavan: 1971. A Global History of the Creation of Bangladesh, S. 68. Auch die indische Regierung wusste darum und im November 1971 sollte Zhou Enlai dies noch einmal betonen, was Bhutto jedoch nicht davon abhielt, kurz darauf öffentlich seine Dankbarkeit für die nicht gegegebene Zusicherung Pekings zu bekunden, man werde im Falle eines indischen Angriffs an Pakistans Seite stehen. S. Sisson / Rose: War and Secession, S. 250-2; Raghavan: 1971. A Global History of the Creation of Bangladesh, S. 203; Guha: India after Gandhi, S. 186.

29 Tatsächlich waren Bengalis die maßgeblichen Triebfedern der pakistanisch-chinesischen Annäherung in den Jahren zuvor gewesen. S. Raghavan: 1971. A Global History of the Creation of Bangladesh, S. 186.

30 Wagner: Die „verhinderte” Großmacht? S. 185; Raghavan: 1971. A Global History of the Creation of Bangladesh, S. 106-7.

31 Raghavan: Between Regional and Global Interests: The Indo-Soviet Treaty of 1971, S. 341; Raghavan: 1971. A Global History of the Creation of Bangladesh, S. 127.

32 McMahon: On the Periphery of a Global Conflict: India and the Cold War, 1947-1991, S. 277. Zum Zusammenhang zwischen Kolonialerfahrung und späterer Bündnisfreiheit, s. Volker Matthies: Die Blockfreien. Ursprünge, Entwicklung, Konzeptionen. Opladen: Leske + Budrich 1985, S. 43. 
1946 hatte der spätere erste Premierminister des unabhängigen Indien, Jawaharlal Nehru, festgestellt: „We shall take full part in international conferences as a free nation with our own policy and not merely as a satellite of another." 33 Übersetzt in das Mächteverhältnis des sich schon damals abzeichnenden Ost-West-Konflikts bedeutete dies aus seiner Sicht: „India will follow an independent policy, keeping away from the power politics of groups aligned one against another.“34 Diese von Nehru formulierte Doktrin der Bündnisfreiheit („non-alignment“) war für Indien wie für die ihm in der „Bewegung Bündnisfreier Staaten“ folgenden Länder elementarer Teil seiner internationalen Sicherheitspolitik und sollte zur Leitlinie aller künftigen indischen Regierungen werden. Sie wollten von der Rivalität der Großmächte profitieren, ohne in sie hineingezogen werden. ${ }^{35}$

Die überraschende chinesisch-amerikanische Annäherung stellte dieses Grundprinzip der indischen Außenpolitik im Sommer 1971 erstmals ernsthaft infrage. Der damalige indische Außenminister Swaran Singh erläuterte vor dem indischen Unterhaus: ,while we welcome a rapprochement between Peking and Washington, we cannot look upon it with equanimity if it means domination of the two countries over this region. " ${ }^{36}$ Als Reaktion wandte sich die indische Regierung an den einzigen Staat, der aus ihrer Perspektive ein wirksames Gegengewicht zur neuen Achse Washington - Peking bilden konnte: die UdSSR.

Ähnlich wie die USA hatte auch die Sowjetunion Ende der 1960er Jahre sowohl Indien als auch Pakistan mit Waffen beliefert. ${ }^{37}$ Aufgrund der traditionellen Allianz Pakistans mit den USA und seiner allmählichen Annäherung an die VR China sympathisierte die Moskauer Führung aber grundsätzlich eher mit Indien. Außerdem teilte sie Indiens Standpunkt im symbolisch außerordentlich bedeutsamen Kaschmir-Konflikt und sah in dem Land eine wichtige Zugangsmöglichkeit zu den anderen bündnisfreien Staaten in Asien und Afrika. ${ }^{38}$

33 Kulwant Rai Gupta / Vatsala Shukla: Foreign Policy of India, Bd. 1. New Delhi: Atlantic Publishers \& Distributors (P) Ltd. 2009, S. 90; vgl. auch McMahon: Heiße Kriege im Kalten Krieg, S. 18. 34 Zit. n. McMahon: On the Periphery of a Global Conflict: India and the Cold War, 1947-1991, S. 277. 35 Matthies: Die Blockfreien, S. 42; Lawrence: The Rise and Fall of Nonalignment, S. 142; Dinkel: Die Bewegung Bündnisfreier Staaten, S. 108-9. ,Even in accepting economic help, it is not a wise policy to put all our eggs in one basket“, lautete Nehrus Empfehlung, zit. n. McMahon: On the Periphery of a Global Conflict: India and the Cold War, 1947-1991, S. 277-8.

36 Zit. n. Barnds: India, Pakistan and the Great Powers, S. 246.

37 Heß: Bangladesch. Tragödie einer Staatsgründung, S. 109; Raghavan: 1971. A Global History of the Creation of Bangladesh, S. 109.

38 Raghavan: 1971. A Global History of the Creation of Bangladesh, S. 3. Im Mai 1969 stellte die sowjetische Führung in Islamabad klar, dass es eine gleichzeitige Partnerschaft mit Peking und Moskau nicht geben würde, s. Matinuddin: Tragedy of Errors, S. 315; Kux: The United States and Pakistan, S. 180-1; Sisson / Rose: War and Secession, S. 238. 
In Neu-Delhi nahm man diese Moskauer Sympathie grundsätzlich wohlwollend zur Kenntnis. Als sowjetische Diplomaten der indischen Regierung im März 1969 den Abschluss eines Freundschafts- und Kooperationsvertrages zwischen den beiden Staaten nahelegten, reagierte Indira Gandhi dennoch zunächst verhalten. Der Vertrag sollte zwar ausdrücklich keine militärische Zusammenarbeit umfassen, aber doch eine wirksame Rückversicherung Indiens gegen etwaige pakistanische oder chinesische Aggression darstellen. Die indische Premierministerin fürchtete den Widerstand sowohl ihrer politischen Opposition als auch aus den eigenen Reihen der Kongresspartei, die eine solche Entfremdung Indiens vom Westen (und damit eine schleichende Abkehr von Indiens bündnisfreier Außenpolitik) nicht hinnehmen würden. Außerdem hoffte Indira Gandhi nach dem Grenzkrieg von 1962 weiterhin auf eine chinesisch-indische Entspannung. ${ }^{39}$

Einen ersten sowjetischen Vertragsentwurf, der langfristig auf die Schaffung eines kollektiven asiatischen Sicherheitssystems zur Eindämmung der VR China abgezielt hätte, lehnte die indische Premierministerin dementsprechend ab. Als sich im Sommer 1969 dann die Spaltung der regierenden Kongresspartei abzuzeichnen begann, wurden die Pläne für den Vertrag vorerst auf Eis gelegt. Indira Gandhi vertröstete ihre sowjetischen Gesprächspartner auf einen Zeitpunkt nach den im Jahre 1971 anstehenden Unterhauswahlen. Sie hoffte, dann über eine komfortablere Mehrheit zu verfügen als in der damaligen Minderheitsregierung. ${ }^{40}$

Anfang der 1970er Jahre bestand die Asienstrategie des sowjetischen Außenministeriums in der Errichtung eines kollektiven Sicherheitssystems unter Moskauer Führung. Vor diesem Hintergrund passte der sich im Frühjahr 1971 andeutende Konflikt zwischen zwei südasiatischen Staaten den sowjetischen Außenpolitikern kaum ins Konzept. Im März 1971 boten sie sich daher als Vermittler im indisch-pakistanischen Streit um die „Ganga“-Entführung und anschlie-

39 Raghavan: 1971. A Global History of the Creation of Bangladesh, S. 109-112.

40 Sisson / Rose: War and Secession, S. 239; Raghavan: 1971. A Global History of the Creation of Bangladesh, S. 113-4; Wagner: Die „verhinderte“ Großmacht? S. 207-8. Die UdSSR blieb derweil jedoch nicht untätig: Im Mai 1969 schlug der stellvertretende sowjetische Ministerpräsident Alexei Kossygin dem neuen pakistanischen Präsidenten Yahya Khan den Abschluss eines TransitHandelsabkommens vor, um auf diesem Wege einen weiteren Zugang nach Südasien zu erhalten. Nur aufgrund von Druck des pakistanischen Außenministeriums und seiner Generalität, die angesichts der sowjetisch-chinesischen Entfremdung um die pakistanisch-chinesische Freundschaft fürchteten, konnte Yahya Khan davon abgehalten werden, diesem Vorschlag Kossygins zuzustimmen. S. Kux: The United States and Pakistan, S. 180. 
ßende Verhängung eines indischen Überflugverbotes gegen Pakistan an. Die indische Regierung lehnte eine solche Vermittlerrolle der UdSSR jedoch ab. ${ }^{41}$

Auch in den Folgemonaten bemühte sich die sowjetische Führung noch, den Konflikt als ein rein innerpakistanisches Problem zu betrachten. ${ }^{42}$ Damit unterschied sie sich in der Bewertung der Situation in Ostpakistan fundamental von der indischen Regierung. Während Letztere eine Änderung der politischen Situation in Ostbengalen als eine unabdingbare Voraussetzung zur Lösung des auch Indien betreffenden Flüchtlingsproblems betrachtete, bestand dieser Zusammenhang nach Ansicht der Moskauer Führung nicht. ${ }^{43}$

Aus indischer Perspektive war das sowjetische Auftreten in diesem Konflikt durchaus widersprüchlich. Nach dem 25. März 1971 forderte Nikolai Podgorny, formelles Staatsoberhaupt der UdSSR, Yahya Khan zwar auf, die Massaker in Ostpakistan $\mathrm{zu}$ beenden und eine friedliche politische Lösung für das Land zu finden, bereits zugesagte Rüstungsgüter lieferte die UdSSR dennoch weiterhin nach Pakistan. Auch Indien versorgte die sowjetische Führung im Frühsommer 1971 mit Rüstungsgütern und erklärte bei einem Besuch des indischen Außenministers Swaran Singh in Moskau am 8. Juni 1971 ihre Solidarität mit der indischen Regierung. ${ }^{44}$ Einem Wunsch Yahya Khans entsprechend wirkte sie dabei jedoch auch beschwichtigend auf die indische Seite ein. So riet der sowjetische Ministerpräsident Alexei Kossygin dem indischen Außenminister, die demokratischen Kräfte in Ostpakistan weiterhin allenfalls indirekt zu unterstützen. ${ }^{45}$ Die Moskauer Führung mahnte also beide Seiten gleichermaßen zur Zurückhaltung.

Als Reaktion auf die amerikanische Annäherung an die VR China beschloss sie allerdings, im Konflikt auf dem indischen Subkontinent klarer Partei zu ergreifen und Indien dadurch stärker an sich zu binden. Da ein gegen Indien gerichtetes Zusammenwirken Pakistans, der VR China und der USA plötzlich realistisch erschien, war nun auch aus indischer Sicht der Moment für den Vertrags-

41 Diehl an AA und Botschaft Rawalpindi sowie Generalkonsulat Dacca, 12. März 1971, PA AA, B 81, Bd. 844; s. auch Sisson / Rose: War and Secession, S. 240-1.

42 Matinuddin: Tragedy of Errors, S. 315; Raghavan: 1971. A Global History of the Creation of Bangladesh, S. 115.

43 Raghavan: Between Regional and Global Interests. The Indo-Soviet Treaty of 1971, S. 331; Raghavan: 1971. A Global History of the Creation of Bangladesh, S. 124.

44 Raghavan: 1971. A Global History of the Creation of Bangladesh, S. 115; Kux: The United States and Pakistan, S. 189; Sisson / Rose: War and Secession, S. 240-1; Guha: India after Gandhi, S. 455; Prasad: Indo-Soviet Relations 1947-1972, S. 378.

45 Raghavan: 1971. A Global History of the Creation of Bangladesh, S. 124. Allerdings setzte man sich auch wiederum dafür ein, linksgerichteten Parteien Posten in der provisorischen Regierung von Bangladesch zu sichern, s. Heß: Bangladesch. Tragödie einer Staatsgründung, S. 78. 
schluss mit Moskau gekommen. ${ }^{46}$ Nachdem Kissinger Mitte Juli 1971 noch einmal unterstrichen hatte, dass Indien im Falle eines Krieges gegen die VR China mit keinerlei US-amerikanischer Unterstützung rechnen könne, wurde der indische Botschafter in Moskau Anfang August 1971 damit beauftragt, die Vertragsdetails auszuhandeln. ${ }^{47}$

Am 9. August 1971 unterzeichneten die Außenminister von Indien und der UdSSR schließlich den Vertrag über „Frieden, Freundschaft und Zusammenarbeit“. Bedeutendste Regelung sollten zwei Sätze in Artikel IX werden, die in früheren Vertragsentwürfen noch gefehlt hatten: ${ }^{48}$

Jede der Hohen vertragschließenden Seiten verpflichtet sich, sich jeglicher Hilfe für eine an einem bewaffneten Konflikt mit der anderen Seite beteiligte dritte Seite zu enthalten. Sollte eine der Seiten Objekt eines Überfalls werden oder sollte ihr ein Überfall drohen, so werden die Hohen vertragschließenden Seiten unverzüglich gegenseitige Konsultationen mit dem Ziel aufnehmen, diese Gefahr zu beseitigen und entsprechende effektive Maßnahmen zur Gewährleistung des Friedens und der Sicherheit ihrer Länder ergreifen. ${ }^{49}$

Da der Regierung Indira Gandhi an der indischen Bündnisfreiheit weiterhin sehr gelegen war, wurde diese im Vertrag ausdrücklich als Basis der gegenseitigen Freundschaft anerkannt. Aus Moskauer Sicht stellte er demnach weniger eine Bindung Indiens an das sozialistische Staatenbündnis dar als eine sicherheitspolitische Rückversicherung, die einen aktiven militärischen Konflikt in Südasien überflüssig machen würde. ${ }^{50}$ Dennoch avancierte Indien mit Abschluss dieses Vertrages zum wichtigsten nichtkommunistischen Partner der UdSSR. ${ }^{51}$ Sowohl die UdSSR als auch Indien bemühten sich in den Tagen nach dem Vertragsschluss zu betonen, dass es keine militärische Lösung für das ostbengalische Flüchtlingsproblem geben könne. ${ }^{52}$ Indira Gandhi hegte gar die Hoffnung, einen ähnlichen

46 Chopra: Ostbengalen - Eine Krise für Indien. Eine Darstellung aus indischer Sicht, S. 615; Wagner: Die „verhinderte“ Großmacht? S. 208; Matinuddin: Tragedy of Errors, S. 315-6; Prasad: Indo-Soviet Relations 1947-1972, S. 376-8; Raghavan: 1971. A Global History of the Creation of Bangladesh, S. 121.

47 Raghavan: 1971. A Global History of the Creation of Bangladesh, S. 128-9; Linde: Bangla Desh. Indien und die Großmächte im Pakistanischen Konflikt, S. 72-74.

48 Sisson / Rose: War and Sessession, S. 198.

49 Zit. n. Linde: Bangla Desh. Indien und die Großmächte im Pakistanischen Konflikt, S. 132; vgl. auch Wagner: Die „verhinderte“ Großmacht? S. 208.

50 Prasad: Indo-Soviet Relations 1947-1972, S. 380; Heß: Bangladesch. Tragödie einer Staatsgründung, S. 86; Raghavan: 1971. A Global History of the Creation of Bangladesh, S. 123.

51 Wagner: Die „verhinderte“ Großmacht? S. 208-9.

52 Heß: Bangladesch. Tragödie einer Staatsgründung, S. 87. 
Freundschaftsvertrag bald auch mit der VR China abschließen zu können. ${ }^{53}$ Obgleich durch den Vertrag im Kriegsfall sowjetische Rüstungsexporte nach Pakistan ausgeschlossen wurden, nahm man ihn auch dort weniger als Bedrohung wahr, denn als einen stabilisierenden Faktor, der einem drohenden militärischen Konflikt vorbeugen sollte. ${ }^{54}$ Und jedes deeskalierende Moment konnte der Konflikt um Ostpakistan zu diesem Zeitpunkt bereits sehr gut gebrauchen.

\section{Der ostbengalische Freiheitskampf wird international}

With the treaty, Moscow threw a lighted match into a powder keg.

Henry Kissinger über den indisch-sowjetischen Freundschaftsvertrag ${ }^{55}$

Am 11. August 1971 wurde der in Westpakistan inhaftierte Mujibur Rahman wegen Hochverrats angeklagt ${ }^{56}$ und einer Initiative Zulfikar Ali Bhuttos folgend disqualifizierte die zentrale pakistanische Kriegsrechtsverwaltung 79 der 160 in die Nationalversammlung gewählten Mitglieder der Awami League. ${ }^{57}$ Eine friedliche Lösung des Konflikts um Ostpakistan rückte damit in weite Ferne.

Indira Gandhi begann daraufhin, über die Mukti Bahini stärkeren militärischen Druck auf die pakistanische Armee auszuüben, um die Zentralregierung zu Verhandlungen mit Mujibur Rahman zu zwingen. Die ostbengalischen Unabhängigkeitskämpfer forderten diese indische Unterstützung inzwischen verstärkt ein, zumal sie im Frühjahr 1971 kaum dazu in der Lage gewesen waren, der pakistanischen Armee nennenswerten Schaden zuzufügen. ${ }^{58}$ Im Spätsommer und Herbst 1971 rüstete die indische Armee die ostbengalischen Guerillakämpfer noch weiter auf und kämpfte immer offener auch Seite an Seite mit ihnen. Erschwert wurde die Zusammenarbeit allerdings dadurch, dass die bengalische Befreiungsbewegung und die Führung der Awami League innerlich zerstritten blieben, sodass die Regierung Indira Gandhi wiederholt vor der Aufgabe stand, sie auf das gemeinsame Ziel der nationalen Unabhängigkeit auszurichten. ${ }^{59}$

53 Raghavan: 1971. A Global History of the Creation of Bangladesh, S. 200.

54 Ebd., S. 125; Heß: Bangladesch. Tragödie einer Staatsgründung, S. 94.

55 Zit. n. Kux: The United States and Pakistan, S. 195.

56 AA / IfZ: $A A P d B$, 1971, Bd. 3, Dok. 389, Anm. 6.

57 Raghavan: 1971. A Global History of the Creation of Bangladesh, S. 207-8; Sisson / Rose: War and Secession, S. 172-3.

58 Bass: The Blood Telegram, S. 185; Raghavan: 1971. A Global History of the Creation of Bangladesh, S. 210-1; Van Schendel: A History of Bangladesh, S. 166.

59 Raghavan: 1971. A Global History of the Creation of Bangladesh, S. 212-5. So galt es im August und September 1971, abtrünnige Politiker der Awami League zur Räson zu rufen, die über das 
Die Großmächte wurden derweil zunehmend zu unsichtbaren Dritten in diesem Konflikt. Die US-Regierung ergriff offen Partei und unterstützte die Kämpfe der mit ihr verbündeten pakistanischen Zentralregierung gegen die Verbündeten ihres politischen Gegners UdSSR. Dadurch machte sie den Bangladeschkrieg zu einem „heißen Krieg “ des Kalten Krieges..$^{60}$ Angesichts der Massaker der pakistanischen Armee an der ostbengalischen Zivilbevölkerung und der offenkundigen strategischen Nachteile der pakistanischen Position auf dem indischen Subkontinent waren einige US-Senatoren um Edward Kennedy weit weniger überzeugt als Nixon und Kissinger, dass die USA mit Pakistan den richtigen Partner im sich andeutenden Dritten Indisch-Pakistanischen Krieg gewählt hatten. Sie verlangten eine radikale Wende in der amerikanischen Südasienpolitik. Die indische Regierung war sich dieser innenpolitischen Uneinigkeit durchaus bewusst und prangerte die pakistanischen Gräueltaten öffentlichkeitswirksam in den USA an. Die freundlich gesinnten US-Senatoren lud sie ein, sich in indischen Flüchtlingscamps persönlich ein Bild von der Lage zu machen. ${ }^{61}$

Mitte August 1971 gelang es diesen US-Politikern schließlich, Nixon zumindest davon zu überzeugen, auf Yahya Khan einzuwirken, die gegen Mujibur Rahman mittlerweile verhängte Todesstrafe nicht zu vollstrecken. Yahya Khan weigerte sich jedoch weiterhin, direkt mit dem inhaftierten Mujibur Rahman $\mathrm{zu}$ verhandeln oder der Awami League ein Treffen mit ihm zu ermöglichen. ${ }^{62}$ Stattdessen ordnete er im September 1971 die Mobilisierung der pakistanischen Streitkräfte im Westflügel des Landes an, woraufhin im Oktober auch die indische Armee ihre Truppen an die jeweiligen Grenzen zu Pakistan zu verlegen begann. ${ }^{63}$

Obwohl Kissinger inzwischen von der Unvermeidbarkeit eines Krieges zwischen Indien und Pakistan überzeugt war und der indisch-sowjetische Freundschaftsvertrag aus US-amerikanischer Sicht bereits im Bewusstsein eines späte-

US-amerikanische Generalkonsulat in direkte Verhandlungen mit dem Regime Yahya Khans in Kontakt zu treten versuchten, s. ebd., S. 218-221.

60 Vgl. Smith: New Bottles for New Wine, S. 582; Greiner: Kalter Krieg und „Cold War Studies.“

61 Matinuddin: Tragedy of Errors, S. 284.

62 Ebd. S. 309; Kux: The United States and Pakistan, S. 196-7. Auf amerikanisches Drängen ersetzte Yahya Khan Ende August 1971 außerdem den Militärgouverneur Ostpakistans, den berüchtigten General Tikka Khan, durch den bengalischen Politiker und ehemaligen Arbeitsminister A. M. Malik, obgleich das Militär weiterhin die Fäden der Macht in Ostpakistan in der Hand behielt. S. ebd., S. 197.

63 Raghavan: 1971. A Global History of the Creation of Bangladesh, S. 222; Sisson / Rose: War and Secession, S. 211. 
ren militärischen Eingriffs Indiens gegen Pakistan geschlossen wurde, ${ }^{64}$ forderte die Nixon-Administration ihre Partner in Islamabad nicht dazu auf, die Anklage gegen Mujibur Rahman zurückzunehmen und mit dem inhaftierten AwamiLeague-Führer zu verhandeln. ${ }^{65}$ Vor allem Präsident Nixon selbst schien die Lage seiner Freunde in der pakistanischen Zentralregierung durchaus persönlich $\mathrm{zu}$ nehmen. Mitte August 1971 verkündete er: „[We] must not - cannot - allow India to use the refugees as a pretext for breaking up Pakistan. “66 Zum größten Verdruss der indischen Regierung setzten die USA sogar ihre Rüstungslieferungen an Pakistan weiter fort. ${ }^{67}$

Die sowjetische Unterstützung für die indische Regierung war demgegenüber weniger umfassend. Zwar stärkte der Vertrag mit der UdSSR der indischen Regierung außenpolitisch zu einem gewissen Grad den Rücken, ${ }^{68}$ doch war die sowjetische Führung deutlich weniger erpicht auf die ostbengalische Unabhängigkeit, als viele in Indien hofften. Aufgrund des großen maoistischen Einflusses in Ostbengalen fürchtete man in Moskau vielmehr die Entstehung eines Vasallenstaates der VR China in Südasien. ${ }^{69}$ Im September 1971 drängten sowjetische Außenpolitiker entsprechend weiterhin beide Seiten zur militärischen Zurückhaltung. ${ }^{70}$

Nach einem Besuch Indira Gandhis in Moskau Ende September 1971 war auch der sowjetischen Führung klar, dass die indische Regierung entschlossen war, militärisch in Ostpakistan einzuschreiten. Nichtsdestotrotz lieferte die UdSSR in den kommenden Wochen noch umfangreich Waffen nach Indien und im Oktober 1971 gelang es der indischen Regierung anlässlich von Konsultationen nach Artikel IX. des Freundschaftsvertrages in Neu-Delhi endlich, die UdSSR von ihrer Einschätzung der Situation in Ostpakistan zu überzeugen. ${ }^{71}$ Zwischen der Sowjetunion und Indien entstand nun eine regelrechte Luftbrücke für Rüstungsgüter. ${ }^{72}$ Ende November 1971 beendete die UdSSR schließlich auch die bis

64 Macmillan: Nixon and Mao, S. 221; AA / IfZ: AAPdB, 1971, Bd. 3, Dok. 451. In diesem Sinne auch Wagner: Die „verhinderte“ Großmacht? S. 208.

65 Ebd., S. $186 .$.

66 Zit. n. Guha: India after Gandhi, S. 455; Kux: The United States and Pakistan, S. 196.

67 Bass: The Blood Telegram, S. 195.

68 Raghavan: 1971. A Global History of the Creation of Bangladesh, S. 74.

69 Ebd., S. 116.

70 Ebd., S. 224-5; Heß: Bangladesch. Tragödie einer Staatsgründung, S. 87-8.

71 Sisson / Rose: War and Secession, S. 242-3; Raghavan: 1971. A Global History of the Creation of Bangladesh, S. 226.

72 Linde: Bangla Desh. Indien und die Großmächte im Pakistanischen Konflikt, S. 79; Matinuddin: Tragedy of Errors, S. 317. 
dahin nach wie vor fließenden Wirtschaftshilfezahlungen an die pakistanische Regierung. ${ }^{73}$ Die Strategie der indischen Premierministerin war aufgegangen.

Die Kämpfe in Ostpakistan hatten sich derweil intensiviert. Seit der zweiten Oktoberwoche 1971 operierte auch die indische Armee selbst auf pakistanischem Territorium, die Regierung Indira Gandhi war jedoch peinlich darauf bedacht, jegliche mediale Berichterstattung hierüber zu dementieren bzw. idealerweise gleich zu unterbinden. ${ }^{74}$ Erst recht galt dies gegenüber internationalen Organisationen: Ende Oktober 1971 behauptete die indische Premierministerin auf einer Pressekonferenz „Indien verberge nichts und habe nichts $\mathrm{zu}$ verbergen. UNBeobachter seien daher überflüssig. “75

Aufgrund des brutalen Vorgehens der pakistanischen Armee gegen die eigene Zivilbevölkerung in Ostpakistan sah die indische Regierung sich im Konflikt mit seinem Nachbarn moralisch auf der richtigen Seite. Die militärische Unterstützung der ostbengalischen Unabhängigkeitsbewegung flankierte Indira Gandhi nun mit Versuchen, auch andere Menschen weltweit von diesem Umstand $\mathrm{zu}$ überzeugen, indem sie Schreckensmeldungen aus Ostbengalen öffentlich anprangerte. ${ }^{76}$ Bei einer mehrwöchigen Reise nach Europa und in die USA verwies sie außerdem auf gemeinsame demokratische Werte, die Pakistan offensichtlich fehlten. Ende November 1971 traf sie damit bei Präsident Nixon jedoch auf taube Ohren. ${ }^{77}$ Er machte der indischen Premierministerin stattdessen noch einmal unmissverständlich klar, dass die USA nicht gegen Yahya Khan ins Feld ziehen würden. Indira Gandhi wiederum hielt wenig von Nixons Vorschlag, die indischen Truppen von der indisch-pakistanischen Grenze abzuziehen. ${ }^{78}$

Obwohl auch in Washington große Zweifel an der Bereitschaft der VR China herrschten, zugunsten Pakistans in einen Krieg gegen Indien einzugreifen, fühlte Präsident Nixon sich der Pekinger Führung zur unbedingten Solidarität mit dem

73 Vermerk von Brendonck, 14. Dezember 1971, PA AA, B 37, Bd. 633.

74 Raghavan: 1971. A Global History of the Creation of Bangladesh, S. 231-2.

75 Diehl an AA und Botschaft Rawalpindi, 21. Oktober 1971, BArch, B 136/6321.

76 Matinuddin: Tragedy of Errors, S. 288; Guha: India after Gandhi, S. 453-4.

77 Guha: India after Gandhi, S. 457. Den Ausdruck „dialogue of the deaf“ prägte in diesem Zusammenhang Kux: The United States and Pakistan, S. 198. Drastisch formulierte Nixon angesichts der zunehmend kaum mehr zu verleugnenden indischen Kriegshandlungen in Ostpakistan: „the Indians have screwed us“, zit. n. Bass: The Blood Telegram, S. 258 und „the Indians have been kicking us in the ass for twenty-five years“, zit. n. Bass: The Blood Telegram, S. 262.

78 Raghavan: 1971. A Global History of the Creation of Bangladesh, S. 228. Yahya Khan hatte sich hierzu bereit erklärt und den Einsatz von UN-Beobachtern zur Überwachung der indischpakistanischen Grenze gefordert, s. Moses: Die Vereinten Nationen, humanitäres Engagement und die Menschenrechte, S. 357. 
gemeinsamen Partner verpflichtet. ${ }^{79}$ Innenpolitisch war man in der VR China vollauf mit der Kulturrevolution sowie zunehmend ernsthaften Drohungen der militärisch deutlich überlegenen UdSSR befasst. ${ }^{80}$ Die indische Regierung erfuhr Mitte November 1971, dass ein Vertreter der VR China Yahya Khan anders als die US-Regierung sogar dazu geraten hatte, Mujibur Rahman freizulassen und mit ihm $\mathrm{zu}$ verhandeln. ${ }^{81}$ Entsprechend versicherte Indira Gandhi Bundesaußenminister Walter Scheel bei einem Besuch in Bonn, die VR China sei zu beschäftigt mit internen Problemen, als dass Indien sie als akute Bedrohung wahrnehmen müsste. ${ }^{82}$

\section{Der „menschliche Preis“}

Die Zahl der Opfer der Massaker der pakistanischen Armee ist nach wie vor umstritten $^{83}$ und im unabhängigen Bangladesch bald zum Politikum geworden. Anhänger der Awami League stilisierten das Morden zur Gründungskatastrophe des Landes, die zu relativieren oder gar infrage zu stellen an Landesverrat grenzte. Nach offiziellen Angaben starben im Kampf um die Unabhängigkeit Bangladeschs drei Millionen Menschen. Wer öffentlich anderes behauptet, riskiert bis heute strafrechtliche Verfolgung. ${ }^{84}$ Nach Regierungsangaben wurden

79 Bass: The Blood Telegram, S. 259.

80 „With Mao’s Cultural Revolution, China virtually ceased to have a foreign policy at all, as its diplomats were summoned home to be cleansed of imperfect attitudes."S. Macmillan: Nixon and Mao, S. xix; Matinuddin: Tragedy of Errors, S. 320. Zu Maos innenpolitischen Herausforderungen im Frühjahr und Sommer 1971, s. Raghavan: 1971. A Global History of the Creation of Bangladesh, S. 201-2.

81 Raghavan: 1971. A Global History of the Creation of Bangladesh, S. 203.

82 Vermerk über ein „Gespräch des indischen Ministerpräsidenten, Indira Gandhi, mit dem Herrn Bundesminister" von Berendonck, 11. November 1971, PA AA, B 37, Bd. 595.

83 Schätzungen gehen von 30.000 bis 3 Millionen. S. Matinuddin: Tragedy of Errors, S. 260; Sarmila Bose: The Question of Genocide and the Quest for Justice in the 1971 War. In: Journal of Genocide Research 13,4 (2011), S. 393-419, hier S. 399-400. Realistisch scheinen letzterer Autorin insgesamt etwa 100.000 Kriegsopfer. S. Bose: Dead Reckoning. Memories of the 1971 Bangladesh War, S. 181.

84 David Bergmann: The Politics of Bangladesh's Genocide Debate. In: The New York Times, 05.04.2016, http://www.nytimes.com/2016/04/06/opinion/the-politics-of-bangladeshs-genocide-debate.html?_r=0 (Zugriff am 08.10.2018); Bose: The Question of Genocide and the Quest for Justice in the 1971 War, S. 394. Dies und die mangelnde Aufarbeitung eigener Kriegsverbrechen an kollaborierenden Bevölkerungsteilen, vor allem den Biharis, ist nach wie vor Nährboden verschiedener Verschwörungstheorien um Wahrheit, Schweigen und nicht zuletzt die Rolle Indiens in diesem Konflikt, an dem sein benachbarter Erzfeind schließlich zerbrechen sollte. S. etwa M. Abdul Mu'min Chowdhury: Behind the Myth of Three Million. London: Al-Hilal Publishers Ltd. 1996. 
zudem 70.000 Bengalinnen infolge von Vergewaltigungen durch die pakistanische Armee schwanger. ${ }^{85}$ Angesichts der Tatsache, dass in diesem Konflikt westpakistanische Armeeangehörige systematisch die bengalische Zivilbevölkerung töteten, war bald von einem Völkermord die Rede..$^{86}$

Die internationale Gemeinschaft blieb angesichts des massenhaften Sterbens in Ostbengalen weitgehend tatenlos ${ }^{87}$ Federführend war hierbei die Regierung der USA, die sich eher um das Schicksal ihres südasiatischen Verbündeten Pakistan im Konflikt mit dem sowjetischen „Vasallen“ Indien sorgte. Dies ist der „menschliche Preis“ des Kalten Krieges: Um ihre schwächeren, aber strategisch wichtigen Verbündeten nicht zu verprellen, nahmen die Großmächte deren Verbrechen hin oder unterstützten mitunter sogar ihr Tun. ${ }^{88}$ Im Falle des ostpakistanischen Bürgerkrieges im Jahre 1971 tat die Nixon-Administration dies, indem sie die pakistanische Armee mit den Waffen ausrüstete, die sie benötigte, um die eigene Zivilbevölkerung zu bekämpfen. Nationalstaatliche Eigeninteressen überwogen im Weißen Haus eindeutig Menschlichkeit und humanitäres Verantwortungsbewusstsein.

Besonders enttäuschend war aus indischer Sicht die Tatsache, dass dies nicht nur für die USA, sondern auch für die meisten afrikanischen und asiatischen Staaten galt, die entweder die pakistanische Zentralregierung unterstützten oder sich einer Positionierung in diesem Konflikt enthielten. Die indische Premierministerin hatte darauf gehofft, in diesen ehemals „Mit-Unterdrückten“, den ehemaligen Kolonien, Verbündete im Kampf gegen die wiederum unterdrückende pakistanische Zentralregierung zu finden. Im Konflikt um Ostpakistan musste sie nun jedoch die Erfahrung machen, dass „das Prinzip der Nichteinmischung über Interventionen zugunsten der Menschenrechte“ triumphierte. ${ }^{89}$

Auch die Bundesregierung hielt sich bedeckt. Dabei war man im Auswärtigen Amt durch Berichte aus dem Generalkonsulat in Dhaka schon im Sommer 1971 recht detailliert über die Situation in Ostpakistan im Bilde:

85 Heß: Bangladesch. Tragödie einer Staatsgründung, S. 74.

86 Sargent: A Superpower Transformed, S. 94.

87 Moses: Die Vereinten Nationen, humanitäres Engagement und die Menschenrechte, S. 340-1; Pai: The 1971 East Pakistan Genocide. A Realist Perspective, S. 9; ähnlich Raghavan: 1971. A Global History of the Creation of Bangladesh, S. 273. Zur Frage, ob es sich dabei um einen Völkermord handelte, s. Bose: The Question of Genocide and the Quest for Justice in the 1971 War, S. 406-9.

88 Greiner: Kalter Krieg und „Cold War Studies.“

89 Moses: Die Vereinten Nationen, humanitäres Engagement und die Menschenrechte, S. 3656; s. auch Raghavan: 1971. A Global History of the Creation of Bangladesh, S. 155-6 bzw. Barnds: India, Pakistan and the Great Powers, S. 243. 
Bei den anhaltenden kill-and-burn-Vergeltungsaktionen auf den Dörfern werden alle Bewohner mit Maschinengewehrgarben auf dem Dorfplatz zusammengetrieben. Wer nicht die für Mohammedaner typischen Beschneidungsmerkmale aufweist oder die fünf Grundsuren des Koran hersagen kann, gilt als verdächtiger Hindu und wird erschossen. ${ }^{90}$

Auch der Bundestagsabgeordnete Hans Edgar Jahn (CDU) gab nach einem Besuch in westbengalischen Flüchtlingslagern Mitte August 1971 an, er begreife „daß das, was hier geschieht, ein Verstoß gegen die Menschenrechtskonvention der UNO und damit ein Verbrechen gegen die Menschlichkeit ist, dem mit allen politischen Mitteln entgegengewirkt werden muss.“ Er versprach, nach seiner Rückkehr nach Deutschland werde er sich „,als Abgeordneter und Publizist nicht nur im Bundestag, sondern auch im Europäischen Parlament dafür einsetzen, daß eine Lösung für dieses Vertreibungsproblem als Verpflichtung für alle Nationen angesehen wird. “91 Allein - seine Stimme sollte ebenso wenig gehört werden wie die der zahlreichen anderen internationalen Zeugen der ostbengalischen Kriegsgräuel. ${ }^{92}$ Die im Herbst 1971 infolge der ausgebrochenen Unruhen und der Zerstörung wichtiger Infrastruktur drohende Hungersnot in Ostpakistan verschärfte die Lage vor Ort zusätzlich. ${ }^{93}$

\subsection{Bundesrepublik, DDR und der Kalte Krieg in Südasien}

Bitter enttäuscht über die Untätigkeit der internationalen Gemeinschaft absolvierte Indira Gandhi im Herbst 1971 eine Reihe von Staatsbesuchen, um im Ausland für ihren Standpunkt im Konflikt mit Pakistan zu werben. ${ }^{94}$ Im November 1971 führte diese Tour die indische Regierungschefin auch in die Hauptstadt der Bundesrepublik Deutschland. Während die Situation auf dem indischen

90 Papenfuß an AA und Botschaft Rawalpindi, 30. Juli 1971, PA AA, B 30, Bd. 673.

91 Kuhna an AA, Botschaft Neu-Delhi sowie Generalkonsulate Bombay und Madras, 12. August 1971, PA AA, B 30, Bd. 673.

92 Es gelang der internationalen Gemeinschaft allerdings, im Laufe des Jahres 1971 immerhin fast 200 Mio. Dollar aufzubringen, um die indische Regierung bei der Versorgung der ostpakistanischen Flüchtlinge zu unterstützen. S. Heß: Bangladesch. Tragödie einer Staatsgründung, S. 83. Außerdem stellte das UN-Flüchtlingshilfswerk (United Nations High Commissioner for Refugees - UNHCR) Unterkünfte für 6,8 Millionen Personen in 896 indischen Lagern. S. Jeanna Kinnebrew / Amy L. Sayward: Making and Keeping the Peace. U.N. Peacekeeping and Refugee Assistance Operations. In: Amy L. Sayward (Hrsg.): The United Nations in International History. London / New York: Bloomsbury 2017, S. 121-146, hier S. 135.

93 Heß: Bangladesch. Tragödie einer Staatsgründung, S. 101-2.

94 Raghavan: 1971. A Global History of the Creation of Bangladesh, S. 226-7. 
Subkontinent eskalierte, wurden die beiden deutschen Staaten ihrerseits zunehmend zu einem wichtigen Schauplatz im Kampf der südasiatischen Konfliktparteien um die internationale Deutungshoheit über den sich nun immer deutlicher abzeichnenden Dritten Indisch-Pakistanischen Krieg.

Anders als in anderen Ländern traf das südasiatische Werben in Bundesrepublik und DDR allerdings auf zwei spezifische und eindeutig unvereinbare Interessen: das Streben der DDR nach internationaler Anerkennung und den Wunsch der Bundesrepublik, eben diese diplomatische Anerkennung der DDR noch möglichst lange hinauszuzögern. Dieses Spannungsfeld prägte auch nach Abschluss des indisch-sowjetischen Freundschaftsvertrages am 9. August 1971 noch sämtliche zwischenstaatliche Interaktionen zwischen den beiden deutschen Staaten und dem indischen Subkontinent.

\section{Der Indisch-Sowjetische Vertrag zwischen Bonn und Ost-Berlin}

Oberstes Ziel ihrer Indienpolitik war aus Sicht der DDR-Führung nach wie vor die diplomatische Anerkennung. Daran sollte sich auch ihr Umgang mit dem aufkommenden Konflikt um Ostpakistan orientieren. Man hatte sich bewusst auf die Seite Indiens gestellt und bemühte sich, dies gegenüber indischen Gesprächspartnern immer wieder herauszustellen. Den indisch-sowjetischen Freundschaftsvertrag vom 9. August 1971 interpretierten DDR-Diplomaten in der Ost-Berliner MfAA-Zentrale vor diesem Hintergrund als einen Schritt der Annäherung Indiens an das sozialistische Staatenbündnis. Der Vertrag setze „neue Maßstäbe für die Beziehungen zwischen der SU und den sozialistischen Ländern einerseits und den antiimperialistischen Entwicklungsländern andererseits“ ${ }^{95}$ hieß es dort. Die Bundesrepublik wiederum versuchte man in Indien durch Hinweise zu diskreditieren, „daß die BRD ,im Rahmen der USA-Globalstrategie eine Schlüsselstellung' einnimmt und dass die BRD ebenso wie die USA Pakistan unterstützt.“96

Bald allerdings sollte die auf den Vertragsabschluss folgende Euphorie in der DDR einen deutlichen Dämpfer erhalten. Zeitgleich mit dem US-amerikanischen Senator Edward Kennedy besuchte Mitte August 1971 auch eine Delegation der Volkskammer der DDR Indien. Sie erfuhr dabei zwar durchaus Dankbarkeit für die

95 Radde an die Leiter der Vertretungen der DDR in Indien, Indonesien, Burma, Ceylon, Kambodscha, 1. September 1971, PA AA, M 1, C 1774/76.

96 „Direktive der Delegation der Volkskammer der DDR für den Aufenthalt in Indien“, Anlage zur Weisung Reder/MfAA an Wenzel/Generalkonsulat der DDR in Indien, 5. August 1971, PA AA, M 1, C 598/76. 
frühen Solidaritätsbekundungen der DDR im Konflikt um Ostpakistan ${ }^{97}$ und der sowjetische Botschafter in Indien wusste $\mathrm{zu}$ berichten, dass die diplomatische Anerkennung der DDR in den sowjetisch-indischen Verhandlungen zum Freundschaftsvertrag immerhin thematisiert worden war. ${ }^{98}$ Indira Gandhi machte der Delegation jedoch auch klar, dass der in der DDR freudig begrüßte Vertrag die Chancen auf eine baldige Anerkennung nicht unbedingt erhöht hatte. Zunächst müssten sich ,die Wellen etwas glätten, die der Vertrag mit der UdSSR international erzeugt hat“, gab die indische Premierministerin zu bedenken. Abgesehen davon waren die Volkskammervertreter mit dem Gespräch aber zufrieden. Man hatte sich soviel zu erzählen, dass die vorgesehene Zeit um zehn Minuten überschritten wurde. „Kennedy musste solange warten“ berichtete das DDR-Generalkonsulat später. ${ }^{99}$

Die Bundesregierung in Bonn wiederum traf der indisch-sowjetische Freundschaftsvertrag zwar überraschend, eine unmittelbare Auswirkung auf die auch für sie so wichtige Frage einer Anerkennung der DDR durch Indien und Pakistan erwartete sie durch ihn aber nicht. In der westdeutschen Botschaft in Pakistan schätzte man ein, dass der Vertrag die Chancen der sozialistischen DDR in Pakistan kaum verbessert haben dürfte. ${ }^{100}$ Auf bundesrepublikanische Diplomaten in Indien wirkte demgegenüber beruhigend, dass Indira Gandhi unmittelbar vor Unterzeichnung des Vertrages einen Westeuropabesuch für Anfang November 1971 angekündigt hatte. ${ }^{101}$ Eine erste Einordnung des Vertrages durch Botschafter Diehl war durchaus optimistisch: ,aus deutscher Sicht fügt sich, auch was den Zeitpunkt betrifft, die Reise gut in das Konzept ein, das die Botschaft [...] zur Frage der Anerkennung der DDR entwickelt hatte.“102

Diehl bewertete die Sorge Indira Gandhis um die indische Bündnisfreiheit im Kalten Krieg nach Abschluss des Vertrages sogar als hilfreich für das bundesrepublikanische Ziel, eine Anerkennung der DDR durch Indien ein wenig hinauszuzö-

97 „Vermerk über ein Gespräch der Volkskammer-Delegation mit Mr. Banerji, Staatssekretär im indischen Außenministerium am 16.8.1971“ von Wenzel, 20. August 1971, PA AA, M 1, C 598/76. 98 „Vermerk über ein Gespräch der Volkskammerdelegation mit dem sowjetischen Botschafter am 15.8.1971“ von Kraus, 20. August 1971, PA AA, M 1, C 598/76.

99 „Vermerk über ein Gespräch der VK-Delegation mit dem Ministerpräsidenten Indiens, I. Gandhi, am 16.8.1971“ von Wenzel, 17. August 1971, PA AA, M 1, C 598/76.

100 Berger an AA, 20. August 1971, PA AA, ISLA, Bd. 8141.

101 Hampe an Staatssekretärsbüro, 13. August 1971, PA AA, B 37, Bd. 598.

102 Diehl an AA, 9. August 1971, PA AA, B 37, Bd. 595. Schon vor Ausbruch der Krise in Ostpakistan hatte die Bundesregierung darauf gehofft, Indira Gandhi bei einem Besuch in Bonn von einer Anerkennung der DDR abbringen zu können. S. Müller an Staatssekretärsbüro, 4. März 1971, PA AA, B 37, Bd. 595. 
gern. ${ }^{103}$ Auch warnte der indische Generalkonsul in Ost-Berlin, J. C. Ajmani, Ende August 1971 vor einer verfrühten Anerkennung der DDR durch seine Regierung: „with so many countries to woo, we should not be suprised if the GDR loses interest in us - after Recognition - and turns to fresh pastures. The leverage we presently enjoy in both Gemanies will be lost if we acted before the time was ripe. "104

Zuletzt schätzte man im AA auch ein, der Vertrag habe zur Beruhigung der angespannten Lage auf dem Subkontinent beigetragen, indem er der innenpolitischen Kritik an der Regierung Indira Gandhi Wind aus den Segeln nahm. Insofern könne von „einer unmittelbaren Kriegsgefahr [...] zur Zeit nicht gesprochen werden." ${ }^{105}$ Direkte Auswirkungen auf die deutsch-deutschen Beziehungen auf dem indischen Subkontinent hatte der indisch-sowjetische Freundschaftsvertrag vom 9. August 1971 also zunächst nicht. Andere südasiatische Interessen spielten in diesen Wochen und Monaten eine weitaus größere Rolle.

\section{Südasiatische Manipulationsversuche und deutsch-deutsche Reaktionen}

Wenige Tage nach Unterzeichnung des indisch-sowjetischen Freundschaftsvertrages sprach der indische Botschafter im Bonner AA vor, um die Bundesregierung zu bitten, bei der pakistanischen Regierung gegen das angekündigte Strafverfahren gegen Mujibur Rahman zu intervenieren. Yahya Khan habe inzwischen die Möglichkeit eines Todesurteils angedeutet. ${ }^{106}$ Die Bundesregierung teilte die indische Befürchtung, dass eine Verurteilung Mujibur Rahmans die angespannte Lage in Pakistan noch weiter verschärfen könnte. Ein AA-Vertreter kam dieser Bitte bald nach und brachte dem pakistanischen Botschafter in Bonn gegenüber die Sorge des Bundeskanzlers über den gegen den inhaftierten Führer der Awami League angestrengten Prozess zum Ausdruck. ${ }^{107}$

103 Diehl an AA, 8. Oktober 1971, PA AA, B 37, Bd. 595. Später suggerierte er außerdem, „Indien könne durch seine Nichtanerkennung der DDR unter Beweis stellen, daß es trotz des Vertrages mit der SU nicht zu einem ,sowjetischen Satelliten' geworden sei.“ S. „Analyse der Aktivitäten der SPD gegenüber Indien“ von Kraus, 5. Juni 1972, PA AA, M 35, ZR 2947/86.

104 J. C. Ajmani an A. R. Venkateswaran/MEA, 27. August 1971, NAI, Ministry of External Affairs, $\mathrm{H} \mathrm{I} / 1012(82) / 71$.

105 Berendonck an Ref. III A 4, 25. August 1971, PA AA, B 57, Bd. 172. Der politisch gewünschten Ausfuhr von Rüstungsmaterial nach Indien und Pakistan kam dieses Argument sehr entgegen. Vgl. Dietrich an BMWF, 1. September 1971, PA AA, B 57, Bd. 167.

106 Vermerk von Berendonck, 12. August 1971, PA AA, B 37, Bd. 629.

107 Berendonck an Botschaft Rawalpindi, 16. August 1971, PA AA, B 37, Bd. 629. Der pakistanische Gesandte beklagte bei dieser Gelegenheit allerdings auch die „Propagandatätigkeit“ in- 
Abgesehen davon waren westdeutsche Diplomaten weiterhin sehr uneins über den Umgang mit dem Konflikt auf dem indischen Subkontinent. Ende August 1971 warnte der Botschafter der Bundesrepublik in Pakistan Norbert Berger erneut vor einem unabhängigen Ostpakistan. Es sei dort mit einem starken kommunistischen Einfluss zu rechnen und Südasien drohe wirtschaftliches und politisches Chaos, falls Pakistan in zwei Teile zerfiele. Seines Erachtens würden außerdem im Falle eines Abzugs der pakistanischen Armee aus Ostbengalen „Hunderttausende Nichtbengalen dem sicheren Tod preisgegeben.“ Botschafter Berger empfahl, die Regierung seines Gastlandes nicht allzu stark unter Druck zu setzen, indem man etwa hohe Anforderungen an eine ,politische Lösung“ für den Konflikt stellte und für die Zahlung weiterer Entwicklungshilfegelder voraussetzte. ${ }^{108}$

Der südasiatischen Öffentlichkeit gegenüber war die Bundesregierung Mitte September 1971 noch peinlich darauf bedacht, keinesfalls den Anschein einer Parteinahme im Konflikt um Ostbengalen zu erwecken. ${ }^{109}$ Sie beschloss, Bergers Empfehlung zu folgen. Entsprechend hieß es nun, für Hilfszahlungen an Pakistan käme es weniger ,auf die Herstellung bestimmter politischer Gegebenheiten, sondern auf wirtschaftliche Erfolgsaussichten“ an. Vorerst sollte gelten, dass „laufende und vertraglich vereinbarte Entwickungshilfevorhaben weitergeführt, neue Verpflichtungen jedoch nicht eingegangen werden.“ Es gab allerdings auch eine Einschränkung: Sollten sich die Gerüchte einer geänderten Haltung Pakistans gegenüber der DDR erhärten, würde die Bundesregierung von diesem Grundsatz abweichen. In einer Weisung an die bundesrepublikanische Botschaft in Pakistan verband die Bonner AA-Zentrale dies mit einer unmissverständlichen Drohung: „Es bestehen keine Bedenken, dies pakistanischen Gesprächspartnern in geeigneter Form nahezubringen." ${ }^{110}$ Auch im Umgang mit Pakistan war die Entwicklungshilfe also noch immer ein probates Mittel der Bundesregierung zur Durchsetzung ihrer Hallstein-Doktrin.

Die Regierung Indiens wiederum erkannte in den spezifischen deutschlandpolitischen Interessen der DDR einen möglichen Hebel, um die Ost-Berliner Führung zu einer noch klareren Positionierung zu ihren Gunsten zu bewegen. Entsprechend bedachte sie die DDR bei ihrer internationalen Werbekampagne.

discher und ostpakistanischer Studenten, die in der Bundesrepublik gegen die pakistanische Zentralregierung agitierten. S. Vermerk von Berendonck, 17. August 1971, PA AA, B 37, Bd. 629.

108 Berger an AA, 30. August 1971, PA AA, B 37, Bd. 629.

109 Berendonck an Botschaften Rawalpindi und Delhi, 14. September 1971, PA AA, ISLA, Bd. 8128.

110 Müller an Botschaften Rawalpindi und Neu-Delhi, 16. September 1971, PA AA, ISLA, Bd. 8141. 
Im September 1971 besuchte der stellvertretende Vorsitzende des indischen Oberhauses, Bhaurao Dewaji Khobragade, die DDR ${ }^{111}$ und Anfang Oktober 1971 folgte ihm eine erste offizielle Delegation indischer Parlamentarier.

DDR-Außenminister Otto Winzer betonte dieser Delegation gegenüber erneut das Eintreten seines Landes für die ostpakistanische Selbstbestimmung. Die DDR habe in dieser Frage von Anfang an eine klare Haltung eingenommen, zu der sie sich auch weiterhin bekenne. Einer Bitte der indischen Delegation, noch weiter in ihrem Sinne international Einfluss zu nehmen, glaubte Winzer allerdings kaum entsprechen zu können, da die DDR keinerlei Beziehungen mit Pakistan unterhalte. Im Übrigen versprach er jedoch weitere Hilfsgüter zur Versorgung der ostbengalischen Flüchtlinge in Indien. ${ }^{112}$ Der Delegationsbesuch aus Indien wurde für die DDR später zumindest insofern zu einem Erfolg, als der Delegationsleiter - der stellvertretende Sprecher des indischen Unterhauses George Gilbert Swell das Fehlen diplomatischer Beziehungen zwischen Indien und der DDR als einen zu überwindenden Anachronismus bezeichnete. ${ }^{113}$

Zur westdeutschen Genugtuung wiederum rechnete etwa zeitgleich der indische Außenminister Swaran Singh die sich andeutende Entspannung in Europa allein der Neuen Ostpolitik der Regierung Willy Brandts an, ohne das Verhältnis Indiens zur DDR überhaupt zu erwähnen. ${ }^{114}$ Und in einem Gespräch mit dem SED-Politbüromitglied Albert Norden erklärte Indira Gandhi Mitte Oktober 1971 zwar, „daß es indischerseits keine prinzipiellen Einwände gegen die diplomatische Anerkennung“ der DDR mehr gebe, ${ }^{115}$ verwies dann aber darauf, das indi-

111 „Bericht über den Aufenthalt des stellv. Vorsitzenden des indischen Oberhauses, B. D. Khobragade, in der Zeit vom 4. bis 12. September 1971 in der DDR“ von Rehmer, 22. September 1971, BArch, DA 1/10044.

112 „Vermerk über die Aussprache des Ministers für Auswärtige Angelegenheiten der DDR, Gen. O. Winzer, mit der indischen Parlamentsdelegation unter Leitung der stellv. Vorsitzenden des Unterhauses, Prof. G.G. Swell, am 8.10.1971“ von Gahlich, 12. Oktober 1971, PA AA, M 1, C 299/75. So verzichtete die DDR in Neu-Delhi anlässlich ihres Nationalfeiertages am 7. Oktober 1971 auf sonst übliche Empfänge und DDR-Generalkonsul Herbert Fischer übergab Indira Gandhi stattdessen einen Scheck über die durch diese Maßnahme eingesparten Mittel. S. „Protokoll über Gespräch Prof. Albert Nordens mit I. Gandhi am 12.10.1971“ von Herbert Fischer, 13. Oktober 1971, PA AA, M 1, C 1752/76. Außerdem bekundete die DDR-Öffentlichkeit in einer Vielzahl von Benefizkonzerten und Demonstrationen ihre Solidarität mit dem ostbengalischen Unabhängigkeitskampf. S. Misra: India and GDR, S. 96.

113 „Bericht über den DDR-Aufenthalt der Delegation des indischen Parlaments unter Leitung des Stellv. Sprechers des Unterhauses, Prof. G. G. Swell, vom 3. bis 10. Okt. 1971“"von Wenzel, 12. Oktober 1971, PA AA, M 1, C 1755/76.

114 Diehl an AA, 12. Oktober 1971, PA AA, B 37 Bd. 598.

115 „Lage auf dem indischen Subkontinent“, 25. November 1971, PA AA, M 35, ZR 1960/79. 
sche Kabinett sei derzeit zu sehr mit dem Konflikt in Ostbengalen beschäftigt, um die Anerkennungsfrage der DDR zu erörtern. ${ }^{116}$ Einige Tage später machte die indische Premierministerin auf einer Pressekonferenz auch noch einmal öffentlich deutlich, dass sie den Zeitpunkt für eine Anerkennung der DDR noch nicht für gekommen hielt. ${ }^{117}$

Ende Oktober 1971 erklärte der indische Außenminister dem westdeutschen Botschafter in Indien Günter Diehl, er sehe keine Möglichkeit mehr für einen direkten Dialog zwischen Indien und Pakistan zur Lösung des Konflikts um die bengalische Flüchtlingskrise. Er bat stattdessen erneut darum, die Bundesregierung möge wirtschaftlichen Druck auf die pakistanische Zentralregierung ausüben, Mujibur Rahman freizulassen und endlich den Wahlsieg der Awami League zu akzeptieren. Diehl lehnte zwar ab, was Swaran Singh allerdings nicht davon abhielt, ihm zu versichern, Indien würde die deutsch-deutschen Verhandlungen sicher nicht durch eine Anerkennung der DDR stören. ${ }^{118}$

\section{Ostbengalische Kontaktversuche}

Auch die Ostbengalen selbst traten den beiden deutschen Staaten gegenüber nun vermehrt in Erscheinung. Als Mujibur Rahman Mitte August 1971 in Westpakistan der Prozess gemacht werden sollte, bat die provisorische Regierung von Bangladesch die DDR in dramatischen Worten um Unterstützung. ${ }^{119}$ In dieser Zeit trat auch Abdus Samad Azad, der spätere erste Außenminister des unabhängigen Bangladesch, an DDR-Diplomaten in Neu-Delhi mit der Bitte heran, von OstBerlin aus möge ein Aufruf an die Welt ergehen, die Unabhängigkeit Banglade-

116 „Protokoll über das Gespräch Prof. Albert Nordens mit I. Gandhi am 12.10.1971, 11.00 bis 11.50 Uhr“ von Herbert Fischer, 13.Oktober 1971. PA AA, M 1, C 1752/76.

117 Diehl an AA und Botschaft Rawalpindi, 21. Oktober 1971, BArch, B 136/6321.

118 Diehl an AA sowie Botschaften London und Rawalpindi, 30. Oktober 1971, PA AA, B 30, Bd. 670. Nichtsdestotrotz war die Bundesregierung bei der Gewährung zusätzlicher humanitärer Hilfe an Indien weiterhin sehr um Ausgewogenheit im Verhältnis zu Pakistan bemüht. S. Hanemann an Bundeswirtschaftsminister, 5. November 1971, BArch, B 102/122288.

119 „Wir appellieren an Sie, Exzellenz, persönlich und unverzüglich zu intervenieren, um diesem schändlichen Akt und der Prozeßfarce Einhalt zu gebieten.“ Abu Sayeed Chowdhury, Sonderbeauftragter der Regierung von Bangla Desh, London, an „den Ministerpräsidenten der Deutschen Demokratischen Republik“, eingegangen am 11. August 1971, PA AA, M 1, C 1045/77. 
schs anzuerkennen. ${ }^{120}$ Das MfAA wollte hierauf zwar nicht explizit reagieren, ${ }^{121}$ appellierte aber in einer öffentlichen Erklärung im SED-Parteiorgan „Neues Deutschland“ an die pakistanische Zentralregierung, „Gerechtigkeit und Humanität walten zu lassen und Sheikh Mujibur Rahman als den gewählten Repräsentanten der überwältigenden Mehrheit seines Volkes zu achten.“122

DDR-Diplomaten in Neu-Delhi erklärten sich auf Bitten des indischen Außenministeriums außerdem dazu bereit, einen offiziellen Vertreter der provisorischen Regierung Bangladeschs zu treffen. Als dieser dann allerdings um Waffenlieferungen zur Beseitigung der „brutalen Herrschaft der pakistanischen Militärjunta“ bat, scheuten sie eine derart offene Parteinahme dann doch. Im Umgang mit der provisorischen Regierung von Bangladesch sei auf unbedingte Diskretion zu achten, erläuterten die DDR-Vertreter später. ${ }^{123}$ Denn obgleich man dort hoffte, die indische Regierung durch Solidarität mit der ostbengalischen Befreiungsbewegung beeindrucken zu können, fürchtete man im MfAA doch auch die Folgen einer allzu eindeutigen Positionierung im anderen südasiatischen Staat. Namentlich stellte sich die Frage: Wie würde die pakistanische Regierung reagieren, wenn die DDR Bangladesch anerkannte? ${ }^{124}$

Abgesehen von ihrem Interesse an einer diplomatischen Anerkennung durch Indien gewann der Konflikt in und um Ostpakistan für die DDR bald auch eine politisch-ideologische Dimension. Ging es ihr offiziell allein um Unterstützung der von der westpakistanischen Zentralregierung unterdrückten Bengalen in der Ausübung ihres Selbstbestimmungsrechts, so suchten DDR-Politiker inoffiziell doch vor allem den Kontakt $\mathrm{zu}$ örtlichen Kommunisten. ${ }^{125}$ Als sich im Sommer und Herbst 1971 abzuzeichnen begann, dass ein Sieg der Awami League noch auf sich warten lassen würde, fällte die Parteiführung der SED intern ein vernichtendes Urteil über den ostbengalischen Unabhängigkeitskampf und seinen Anführer Mujibur Rahman. Sie betrachtete die Politik der Awami League als einen Versuch

120 Herbert Fischer: Entwicklung der staatlichen und gesellschaftlichen Beziehungen DDR Indien. In: Joachim Heidrich (Hrsg.): DDR - Indien: Partner auf Zeit. Erfahrungen und Einsichten. Hamburg: Lit-Verlag 1998, S. 24-46, hier S. 41; Fischer: DDR - Indien. Ein Diplomat berichtet, S. 69.

121 Kiesewetter an das Sekretariat des Vorsitzenden des Ministerrates z.Hd. des Genossen Böthling, 12. August 1971, PA AA, M 1, C 1045/77.

122 Erklärung eines Sprechers des MfAA der DDR (veröffentlicht am 14. August 1971 im Organ des Zentralkomitees der SED, „Neues Deutschland“), PA AA, M 35, ZR 2949/89.

123 „Vermerk über ein Gespräch mit Mr. Alam, Vertreter der Regierung ,Bangla Desh', am 17.8.1971“ von Kraus, 20. August 1971, PA AA, M 1, C 598/76.

124 Voigt: Die Indienpolitik der DDR, S. 654.

125 Ebd., S. 648-9. 
der „ostpakistanischen Bourgeoisie, mit Hilfe der Werktätigen sich an der Ausbeutung im Gesamtrahmen Pakistans zu beteiligen.“ Mujibur Rahman bewertete sie als „bürgerlichen Bankrotteur [...], der nicht fähig ist, die Interessen der ostpakistanischen Bevölkerung zu sichern.“ Die Einrichtung der provisorischen Regierung von Bangladesch sei nichts als „eine Farce, die zu spät und unter illusionären bürgerlichen Vorstellungen geschah. “Wichtiger als die Unabhängigkeit Ostpakistans waren für den SED-Staat demnach „die entscheidenden strategischen Interessen der sozialistischen Staatengemeinschaft“ unter Führung der Sowjetunion. ${ }^{126}$

Als ein Vertreter des Zentralkomitees der SED Anfang Oktober 1971 auf dem 9. Parteitag der Kommunistischen Partei Indiens im südindischen Cochin vom Generalsekretär der Kommunistischen Partei Ostpakistans, Abdus Salam, um Hilfe im Kampf gegen die pakistanische Zentralregierung gebeten wurde, verwies man entsprechend darauf, er müsse sich in dieser Frage zunächst mit der Kommunistischen Partei der Sowjetunion (KPdSU) abstimmen, bevor die SED tätig werden könne. Ein Vertreter der KPdSU wiederum wies den Besucher aus der DDR bei der Gelegenheit darauf hin, dass die Kommunistische Partei Ostpakistans über lediglich 750 Mitglieder und damit kaum über nennenswerte politische Macht verfügte. ${ }^{127}$ Wenn die DDR-Führung ihre Unterstützung für die ostbengalische Unabhängigkeitsbewegung als Hebel zur Durchsetzung einer Anerkennung durch Indien einsetzen wollte, musste sie sich also mit der Awami League arrangieren.

Die Bundesrepublik wiederum wurde von den Vertretern Ostbengalens erst später als potenzieller Ansprechpartner wahrgenommen. Eine standardisierte Mitteilung über die Eröffnung einer Mission der provisorischen Regierung von Bangladesch in Neu-Delhi ignorierte die dortige westdeutsche Botschaft Ende August 1971. ${ }^{128}$ Am 22. Oktober 1971 nahm man dann zum ersten Mal direkt Kontakt mit der Bundesregierung auf. Der in Abwesenheit Mujibur Rahmans das Amt des Präsidenten von Bangladesch ausübende Syed Nazrul Islam gratulierte Willy Brandt zur Verleihung des Friedensnobelpreises. ${ }^{129}$ Auch dieser Annäherungsversuch gegenüber der Bundesrepublik blieb allerdings folgenlos. Während die DDR-Diplomaten mit den Vertretern des werdenden Bangladesch also längst

126 „Konzeption für ein Gespräch mit der KP Ostpakistans während des IX. Parteitages der KP Indiens“, 30. September 1971, BArch, DY 30/97815.

127 „Vermerk über das Gespräch des Genossen Martin Fischer, Mitglied des ZK der SED, mit dem Generalsekretär der KP Ostpakistan, Genossen Abdus Salam, am 7.10.1971 in Cochin“, 27. Oktober 1971, PA AA, M 1, C 1053/77.

128 Diehl an AA und Botschaft Islamabad, 6. September 1971, PA AA, ISLA, Bd. 8128.

129 Syed Nazrul Islam an Willy Brandt, 22. Oktober 1971, PA AA, B 37, Bd. 583. 
bekannt waren, ließen ihre Gegenspieler aus dem AA die ostbengalischen Kontaktversuche leichtfertig außer Acht.

\section{Indira Gandhi reist nach Bonn}

Von 10. bis 12. November 1971 besuchte schließlich auch die indische Premierministerin Indira Gandhi selbst die Bundesrepublik Deutschland, um für ihren Standpunkt im indisch-pakistanischen Konflikt zu werben. Noch vor ihrem Eintreffen wollte auch der pakistanische Botschafter in Bonn, Jamshed G. Kharas, den Bundeskanzler sprechen. ${ }^{130}$ Am 8. November 1971 erhielt Kharas Gelegenheit dazu und bat Willy Brandt darum, mäßigenden Einfluss auf die indische Premierministerin auszuüben, was dieser zu tun versprach. Der pakistanische Botschafter teilte Brandt mit, Indira Gandhi habe erklärt, sie würde die vielen ostpakistanischen Flüchtlinge nur „nach Bangladesch“ zurückkehren lassen, was für die pakistanische Zentralregierung eine absolut inakzeptable Forderung sei. Er beschwor außerdem das Horrorszenario eines (nach der Teilung von 1947) wiedervereinigten Bengalens unter kommunistischer Regierung herauf. Statt hierauf einzugehen, forderte Brandt die pakistanische Zentralregierung zum Dialog mit dem inhaftierten Mujibur Rahman auf. ${ }^{131}$ Jahrzehnte zuvor hätten doch auch die Briten mit den führenden Kämpfern der indischen Unabhängigkeitsbewegung (Mahatma Gandhi und Jawaharlal Nehru) verhandelt. Dieses Argument wiederum ließ Kharas nicht gelten. Er hielt das Tischtuch zwischen Yahya Khan und Mujibur Rahman für endgültig zerschnitten. ${ }^{132}$

Die indische Premierministerin bestätigte dann jedoch Willy Brandts Einschätzung. Sie versicherte, für eine friedliche Lösung des innerpakistanischen Konflikts brauche es lediglich direkte Verhandlungen der pakistanischen Zentralregierung mit den gewählten Vertretern der Awami League. ${ }^{133}$ In ihrem ersten vertraulichen Gespräch mit dem Bundeskanzler am 10. November 1971 war Indira Gandhi außerdem sehr bemüht, ihre Regierung im Konflikt um Ostpakistan als

130 Vermerk von Müller, 21. Oktober 1971, PA AA, B 37, Bd. 635.

131 Ein Vermittlungsversuch, für den Mujibur Rahman Willy Brandt später ausgesprochen dankbar war, s. AA / IfZ: $A A P d B, 1972$, Bd. 1, Dok. 10.

132 AA / IfZ: AAPdB, 1971, Bd. 3, Dok. 385; „Vermerk über ein Gespräch des Bundeskanzlers mit dem pakistanischen Botschafter“ von Schilling, PA AA, B 1, Bd. 478, 8. November 1971; Vermerk von Schilling, 8. November 1971, PA AA, B 37, Bd. 635.

133 Vermerk über ein „Gespräch des indischen Ministerpräsidenten, Indira Gandhi, mit dem Herrn Bundesminister" von Berendonck, 11. November 1971, PA AA, B 37, Bd. 595; Heimsoeth an Botschaft Islamabad, 12. November 1971, PA AA, ISLA, Bd. 8129. 
lediglich reagierend darzustellen. Sie beteuerte, dass die vielen ostbengalischen Flüchtlinge in Indien ,ein Element der sozialen Unsicherheit und Unruhe“ darstellten. „Man wisse nicht, wieviele Agenten sich darunter befänden.“134

Indira Gandhi betonte, ihre Regierung stünde vor diesem Hintergrund unter einem enormen Druck durch die eigene Bevölkerung. Überhaupt unterstütze die indische Armee die ostbengalischen Guerillas nur minimal. Auf Vorschläge Brandts, die indische Premierministerin möge direkt mit Yahya Khan sprechen, oder aber um ein UN-Mandat werben, ging Indira Gandhi hingegen nicht ein. Stattdessen erwähnte sie die zunehmend lauter werdenden Stimmen innerhalb des indischen Parlaments zugunsten einer Anerkennung der DDR. Zwar wolle man der ersten sozialdemokratischen Regierung in Deutschland seit der Weimarer Republik keine Unannehmlichkeiten bereiten, ${ }^{135}$ doch sei ein gewisses indisches Entgegenkommen der DDR gegenüber notwendig und im Übrigen die Anerkennung durch einen außereuropäischen Staat für das deutsch-deutsche Verhältnis wohl ohnehin kaum besonders relevant. Brandt widersprach vehement: Jegliche moralische Unterstützung der DDR erschwere die aktuellen deutsch-deutschen Verhandlungen. Bevor andere Staaten wie Indien die DDR anerkannten müsse zuerst jedenfalls die Berlin-Frage und das innerdeutsche Verhältnis geregelt sein. Außerdem sei die Vertretung der DDR in Neu-Delhi doch jetzt schon größer als die der Bundesrepublik. ${ }^{136}$

Bereits während dieses ersten Treffens der beiden Regierungschefs wurde also deutlich, was die indische Premierministerin vom westdeutschen Bundeskanzler verlangte und umgekehrt. Am Folgetag bat Indira Gandhi Willy Brandt darum, sich bei Yahya Khan für die Freilassung Mujibur Rahmans einzusetzen,

134 AA / IfZ: $A A P d B, 1971$, Bd. 3, Dok. 389.

135 Ebd. Hierzu hieß es in einem Sachstand zu den bilateralen Beziehungen: „ein Teil der indischen Oberschicht ist vom englischen Sozialismus beeinflußt (London School of Economics); er hat ein Geschichtsbewusstsein übernommen, in dem das kaiserliche und nationalsozialistische Deutschland die Rolle des Störenfrieds spielen; es ist der indischen Linkspresse gelungen, einem Teil der indischen Öffentlichkeit einzusuggerieren, daß die Bundesrepublik Deutschland das ideologische Erbe dieser Staaten [Kaiserreich und Drittes Reich] übernommen hat und daß die Gesellschaft in der Bundesrepublik Deutschland sich nicht nach dem Krieg regenerieren konnte. Gerade aus diesem Grunde hatte die neue Bundesregierung in Indien eine besonders gute Presse: weitgehend erblickte man in ihrer Bildung den Anfang dieser bisher vermißten Regenerierung. “S. Gesprächsmappe zum Besuch Indira Gandhis in Bonn, 22. Oktober 1971, BArch, B 136/6321. Im MfAA der DDR glaubte man später, eine „unbestreitbare, wenn auch mittelbare ideologische Affinität zwischen den Führungskräften der Kongreßpartei und den Politikern der SPD“ zu erkennen. S. „Analyse der Aktivitäten der SPD gegenüber Indien“ von Kraus, 5. Juni 1972, PA AA, M 35, ZR 2947/86.

136 AA / IfZ: $A A P d B, 1971$, Bd. 3, Dok. 389. 
was dieser zu tun versprach. Der indische Gast wiederum wurde gebeten, die DDR zum gegenwärtigen Zeitpunkt noch nicht anzuerkennen. Man versuchte dabei, die deutsch-deutschen Verhandlungen in den globaleren Kontext der Détente im Ost-West-Konflikt einzuordnen. Die „Normalisierung zwischen den beiden deutschen Staaten“ sei Voraussetzung für „breite Projekte der Zusammenarbeit und der Entspannung“ weltweit. Insofern sei die Neue Ostpolitik nichts weniger als ein „Versuch, aus der Geschichte des 20. Jahrhunderts zu lernen.“"137

Die indische Seite ließ sich hiervon weniger beeindrucken als erhofft. Indira Gandhi erkundigte sich zwar nach dem aktuellen Stand der deutsch-deutschen Verhandlungen, ihr Berater P. N. Haksar machte aber auch darauf aufmerksam, dass die westdeutsche Entwicklungshilfe an Indien in den letzten Jahren dramatisch zurückgegangen sei, während die Zahlungen an Pakistan im selben Zeitraum erhöht worden seien. Außerdem erwähnte er zuvor vereinbarte Rüstungsexporte, die nun von westdeutscher Seite überraschend doch wieder gestoppt worden waren. Brandt versprach daraufhin, dem Bundessicherheitsrat die Frage zur erneuten Entscheidung vorzulegen. ${ }^{138}$ Auf seine indischen Gesprächspartner wirkte diese Geste beruhigend und auch die Bonner Zusage von Kapitalhilfe in Höhe von 270 Mio. DM für das Jahr 1972 verfehlte ihre Wirkung nicht. ${ }^{139}$ Selbst DDR-Diplomaten mussten später anerkennen, dass es dem Bundeskanzler durch dieses und ähnliche Manöver gelungen war, „eine antiwestdeutsche Welle in der ind. Öffentlichkeit (wie z.Z. des ind.-pak. Krieges 1965) zu vermeiden.“140

Zwar glaubte Willy Brandt nach seinen Gesprächen mit Indira Gandhi nicht mehr, dass sie einem Krieg mit Pakistan wirklich noch aus dem Weg gehen wollte. ${ }^{141}$ Dennoch gab die Bundesregierung wenig später dem indischen Drängen nach, das gegen sie gerichtete Embargo auf Rüstungslieferungen teilweise aufzuheben, während der gegen Pakistan ebenfalls verhängte Auslieferungsstopp aufrechterhalten blieb. ${ }^{142}$ Brandt wurde damit zum größten Unterstützer Indira Gandhis in Westeuropa. ${ }^{143}$ Im AA war man sich daraufhin sicher, Indira Gandhi

137 AA / IfZ: $A A P d B, 1971$, Bd. 3, Dok. 391.

138 Ebd.

139 Voigt: Die Indienpolitik der DDR, S. 647.

140 „Jahresbericht des Generalkonsulats der DDR in Indien“ von Herbert Fischer, 28. Dezember 1971, PA AA, M 1, C 1728/76.

141 Vermerk über ein „Gespräch des indischen Ministerpräsidenten, Indira Gandhi, mit dem Herrn Bundesminister“"von Berendonck, 11. November 1971, PA AA, B 37, Bd. 595.

142 Das Gupta: India and Ostpolitik, S. 172-3; vgl. auch oben, Abschnitt 2.2.

143 Bass: The Blood Telegram, S. 243. 
würde ihren Besuch in Bonn als Erfolg verbuchen. ${ }^{144}$ Andererseits hatte die indische Delegation zugestimmt, die Bundesregierung vor etwaiger Anerkennung der DDR zu konsultieren, sodass man auch in Bonn zufrieden sein konnte. ${ }^{145}$

Noch während Indira Gandhi sich in Bonn aufhielt, kündigte auch der Staatssekretär im pakistanischen Außenministerium (und während der Kriegsrechtsverwaltung de facto Außenminister), Sultan Khan, seinen Besuch in der Bundesrepublik Deutschland an, um mit einer Gegendarstellung für den pakistanischen Standpunkt im Konflikt um Ostbengalen zu werben. ${ }^{146}$ Tatsächlich traf er am 22. November 1971 den AA-Staatssekretär Paul Frank und bat unter Hinweis auf die Abhängigkeit Pakistans von Rüstungsimporten um die Lieferung westdeutscher Waffenteile nach Pakistan. Mit dem (falschen) Verweis auf die Tatsache, dass Indien in dieser Frage auch noch nicht nachgegeben würde, hielt Frank ihn jedoch hin. ${ }^{147}$

\section{Auf der Schwelle zum Krieg}

Selbst für unbeteiligte Dritte wurde der Konflikt um Ostpakistan inzwischen äußerst gefährlich. Kurz nach Indira Gandhis Abreise aus Bonn forderte er seine ersten und einzigen deutschen Opfer. Am 14. November 1971 hatten zwei Mitarbeiter des westdeutschen Generalkonsulats in Dhaka die Stadt auf eigene Faust mit dem Auto verlassen, um einige ostbengalische Sehenswürdigkeiten zu besichtigen. Zwanzig Kilometer südöstlich der ostpakistanischen Hauptstadt fuhren sie über eine Antipanzermine, die zündete und ihr Fahrzeug durch die Luft in einen Wassergraben schleuderte. Beide waren sofort tot. ${ }^{148}$

Auch wenn der bevorstehende Kriegsausbruch nun kaum mehr zu übersehen war, setzte die Bundesregierung weiter auf Vermittlung. So versuchte Bundesaußenminister Walter Scheel Ende November 1971 vergeblich, seinen sowjetischen Amtskollegen Andrei Gromyko von dessen mit Indien gemeinsam vorgebrachter Forderung abzubringen, die pakistanische Zentralregierung solle vor etwaigen Verhandlungen mit der Awami League zunächst Mujibur Rahman aus der Haft entlassen. Analog zur Argumentation Willy Brandts gegenüber Indira Gandhi

144 Berendonck an Botschaften Delhi, Rawalpindi, Washington, Paris, London, Moskau, Tokio sowie SV bei NATO in Brüssel und UNO in New York, 12. November 1971, PA AA, ISLA, Bd. 8129. 145 Heimsoeth an Botschaft Rawalpindi, 12. November 1971, PA AA, ISLA, Bd. 8129.

146 Vermerk von Müller, 11. November 1971, PA AA, B 8, Bd. 1580.

147 Vermerk von Jungfleisch, 24. November 1971, PA AA, B 37, Bd. 634.

148 „Pressemitteilungen und Sprachregelungen des Pressereferats“ im AA, 18. November 1971, PA AA, B 84, Bd. 840. 
verwies Scheel darauf, dass auch Mahatma Gandhi und Nehru im Gefängnis gesessen hätten, als sie mit den Briten über die Unabhängigkeit Indiens verhandelten. Gromyko ließ dies jedoch nicht gelten. ${ }^{149}$

Im Ost-Berliner MfAA verfolgte man den südasiatischen Konflikt demgegenüber weiterhin allein unter dem Gesichtspunkt der Anerkennungsfrage. Kurz vor Ausbruch des Dritten Indisch-Pakistanischen Krieges war das Bild hier durchwachsen. Obwohl es aus Südasien auch positive Signale wie erneute Annäherungsversuche der pakistanischen Regierung gab, ${ }^{150}$ war die indische Seite seit dem Besuch Indira Gandhis in Bonn der DDR gegenüber wesentlich vorsichtiger. So betonte der indische Generalkonsul in Ost-Berlin, J. C. Ajmani, Ende November 1971 im Gespräch mit DDR-Diplomaten, Indien müsse mit der Anerkennung der DDR noch ,abwarten, um nicht durch so einen Schritt eine scharfe Reaktion (z.B. Waffenlieferungen der BRD an Pakistan) hervorzurufen." Vor diesem Hintergrund könne die Anerkennung nicht vor Abschluss der Verhandlungen zwischen den beiden deutschen Staaten bzw. vor Ende des Konflikts um Ostbengalen erfolgen. ${ }^{151}$ Andererseits wurden DDR-Diplomaten von ihren sowjetischen Partnern davor gewarnt, den pakistanischen Avancen allzu offen $\mathrm{zu}$ begegnen. $\mathrm{Zu}$ leicht könnte sonst die indische Seite verprellt werden. Eine stärkere Parteinahme der DDR-Presse zugunsten Indiens wiederum würde dort vermutlich sehr positiv aufgenommen, hieß es in Moskau. ${ }^{152}$

Die Ost-Berliner DDR-Führung setzte diese gut gemeinten sowjetischen Ratschläge schon bald um. Trotz der ernüchternden Mitteilung Ajmanis und obwohl auch die DDR-Diplomaten selbst nicht daran zweifelten, „daß sowohl in Pakistan als auch in Indien chauvinistische und revanchistische Forderungen extremistischer Kräfte zunehmen, die einen Krieg als ,einzige Lösung’ provozieren

149 AA / IfZ: $A A P d B, 1971$, Bd. 3, Dok. 416.

150 Das pakistanische Außenministerium hatte den dortigen sowjetischen Botschafter informiert, Pakistan sei zu „Verhandlungen über die Frage der Herstellung offizieller staatlicher Beziehungen zur DDR bereit." S. Radde an die Leiter der Vertretungen in New Delhi, Djakarta, Rangoon, Colombo, Phnom Penh, 1. Dezember 1971, PA AA, M 1, C 1774/76.

151 „Vermerk über ein Gespräch des Genossen Dr. Radde mit dem Generalkonsul Indiens in der DDR, Botschafter J.C. Ajmani, am 26.11.1971 im MfAA“ von Rehmer, 30. November 1971, PA AA, M 1, C 1736/76. Ajmani empfand das Drängen der DDR auf Anerkennung mittlerweile als reichlich übertrieben: „GDR policy makers appear to have come to the sad conclusion that India was not to be hustled unless fear was put into her heart that any undue delay might expose her to embarassment“, schrieb er in einem Bericht nach Neu-Delhi. S. J. C. Ajmani an A. R. Venkateswaran/ MEA, 11. November 1971, NAI, Ministry of External Affairs, H I/1012(82)/71.

152 „Vermerk über ein Gespräch zwischen Gen. Lützkendorf und Gen. Fomin, Leiter der Abt. Südasien, am 12.11.1971 im MID“ von Elsner, 16. November 1971, PA AA, M 1, C 1253/77. 
wollen“, ${ }^{153}$ bezog man am 29. November 1971 zum ersten Mal öffentlich klar Stellung zugunsten der indischen Regierung. In einer Regierungserklärung gab der DDR-Ministerratsvorsitzende Willi Stoph der pakistanischen Zentralregierung nunmehr die Hauptschuld an dem seit Monaten schwelenden militärischen Konflikt. ${ }^{154}$

\subsection{Zwischenbilanz}

Im Grunde hatte keine der Großmächte Interesse an einer Eskalation des Konflikts in und um Ostpakistan. Bis zum Ausbruch des Dritten Indisch-Pakistanischen Krieges Anfang Dezember 1971 hoffte man in Washington und Moskau vielmehr weiterhin auf eine politische Lösung und versuchte, die indische Regierung zur Zurückhaltung zu bewegen. Auch die Pekinger Führung war angesichts der wiederholten Beschwichtigungsversuche der beiden Supermächte noch im Oktober 1971 zuversichtlich, dass es keinen neuerlichen Krieg in Südasien geben würde. ${ }^{155}$

Die existenzielle Sorge um seinen südasiatischen Verbündeten Pakistan ließ den US-Präsidenten Richard Nixon dann aber bald klar Stellung zugunsten der pakistanischen Zentralregierung beziehen. Ging es ihm und seinem Sicherheitsberater Kissinger zu Beginn des Bürgerkrieges in Ostpakistan im Frühjahr und Sommer 1971 noch vornehmlich um den Schutz der durch Yahya Khan zu vermittelnden Annäherung an die VR China, so fürchteten die beiden US-Politiker im Herbst 1971 um ihre internationale Glaubwürdigkeit vor allem gegenüber der Pekinger Führung, falls der befürchtete indische Sieg tatsächlich das Ende ihres gemeinsamen Verbündeten Pakistan bedeuten sollte.

Die VR China wurde dadurch im Vorfeld des Bangladeschkriegs zum Zünglein an der Waage des Ost-West-Konflikts. Das Zerwürfnis zwischen der politischen Führung in Peking und ihren sowjetischen Gegenspielern eröffnete der US-Administration um Nixon und Kissinger 1971 die Möglichkeit, sich dem zweitgrößten kommunistischen Staat ohne Gesichtsverlust zuzuwenden. Und für die politische Führung der VR China bedeutete der US-amerikanische Schritt in ihre Richtung einen unschätzbaren Zugewinn an weltpolitischer Bedeutung: Die Regierung in Peking wurde nunmehr international akzeptiert und verdrängte die

153 Radde an die Leiter der Vertretungen in New Delhi, Djakarta, Rangoon, Colombo, Phnom Penh, 1. Dezember 1971, PA AA, M 1, C 1774/76.

154 Singh: Bangla Desh Documents, Bd. 2, S. 215.

155 Matinuddin: Tragedy of Errors, S. 314; Raghavan: 1971. A Global History of the Creation of Bangladesh, S. 200. 
bis dahin im Westen als legitime Vertretung Chinas anerkannte Exilregierung in Taiwan in die politische Bedeutungslosigkeit. Sie würde sogar deren ständigen Sitz im UN-Sicherheitsrat einschließlich des mächtigen Vetorechts übernehmen.

Durch die zwischen der VR China und den USA bzw. Indien und der UdSSR im Sommer 1971 überraschend geknüpften freundschaftlichen Bande war der Frontverlauf des globalen Kalten Krieges auf dem indischen Subkontinent ab Mitte August 1971 klar zementiert. Irgendwo zwischen Islamabad und Neu-Delhi kreuzten sich die Achsen Westpakistan - China - USA einerseits und Ostpakistan - Indien - UdSSR andererseits. ${ }^{156}$ Ein Appell des damaligen UN-Generalsekretärs U Thant verdeutlicht die sicherheitspolitische Brisanz dieser neuen Konstellation: Bereits Anfang August 1971 warnte er den UN-Sicherheitsrat, ein erneuter indisch-pakistanischer Konflikt „could all too easily expand.“157

Bundesrepublik und DDR waren deutlich freier von diesen globalen machtpolitischen Erwägungen, die die politischen Führungen in Washington und Moskau umtrieben. Mit ihren gegensätzlichen deutschlandpolitischen Ambitionen sahen die Außenpolitiker in Bonn und Ost-Berlin im indisch-sowjetischen Freundschaftsvertrag in erster Linie das, was sie darin gerne erkennen wollten. Nach Einschätzung der Bundesregierung hatte Indien sich durch den Abschluss des Freundschaftsvertrages mit der UdSSR bereits allzu weit von seiner bündnisfreien Außenpolitik entfernt. Damit bestand für die indische Regierung aus westdeutscher Sicht kaum mehr Spielraum für eine noch weitergehende Annäherung an das sozialistische Staatenbündnis, indem sie etwa dem Drängen der DDR auf diplomatische Anerkennung nachgab. In der DDR wiederum betrachtete man den Freundschaftsvertrag als das langersehnte Bekenntnis einer indischen Regierung zum sozialistischen Staatenbündnis. Dass es vor diesem Hintergrund bald zur erwünschten Anerkennung kommen musste, war für DDR-Außenpolitiker im Herbst 1971 beständige Hoffnung und Gewissheit.

Die Regierung Indira Gandhi wie auch die pakistanische Zentralregierung waren sich der deutsch-deutschen Partikularinteressen im Herbst 1971 deutlich bewusst und versuchten sie in ihrem Sinne zu nutzen. Wichtiges Mittel zu diesem Zweck war die Entsendung von Politikern und Regierungsvertretern nach Bonn bzw. Ost-Berlin, um für die jeweils eigene Position in diesem bilateralen Konflikt $\mathrm{zu}$ werben. Unter Anleitung der indischen Regierung unternahmen bald auch die offiziellen Vertreter Ostbengalens derartige Versuche.

Während die DDR-Führung auf jedes Zeichen der Annäherung der indischen Regierung sofort reagierte und sich selbst der „Provisorischen Regierung von

156 Vgl. Van Schendel: A History of Bangladesh, S. 169.

157 Zit. n. Barnds: India, Pakistan and the Great Powers, S. 247. 
Bangla Desh“ gegenüber aufgeschlossen zeigte, die doch mangels eigenen Staates noch nicht einmal diplomatische Anerkennung versprechen konnte, wurden entsprechende Signale aus Islamabad wiederholt ignoriert. Geradezu verbissen klammerte sich die DDR-Führung an die Hoffnung auf eine baldige Anerkennung durch Indien und übersah dadurch mehrere gute Gelegenheiten, rasche Erfolge beim anderen südasiatischen Staat zu erzielen. Andererseits wiederum knüpfte sie im Gegensatz zur Bundesregierung schön früh erste Kontakte mit der späteren Regierung von Bangladesch. Ein Vorteil, der sich in den kommenden Monaten noch auszahlen sollte.

Außerdem verfügte die Bundesrepublik Deutschland im Gegensatz zur DDR bereits seit langem über gefestigte Beziehungen $\mathrm{zu}$ beiden verfeindeten südasiatischen Staaten. Daher hatte Indira Gandhi gegenüber dem westdeutschen Bundeskanzler Willy Brandt bei ihrem Bonn-Besuch Anfang November 1971 ein Druckmittel in der Hand, das die Vertreter Indiens bei Reisen in die DDR nicht besaßen: Die indische Premierministerin konnte damit drohen, die DDR anzuerkennen, sollte die Bundesregierung ihrem Wunsch nicht entsprechen, Indien wichtige Rüstungsgüter zu liefern, während das Embargo gegenüber Pakistan aufrechterhalten bleiben sollte.

Indira Gandhi setzte dieses Mittel ausgesprochen wirksam ein und zwang Willy Brandt so gegen seinen Willen zu einer faktischen Ungleichbehandlung der beiden südasiatischen Kontrahenten. Obwohl die pakistanische Zentralregierung die deutschlandpolitischen Interessen der Bundesregierung genauso hätte ausnutzen können, wurde diese Möglichkeit vom pakistanischen Chefdiplomaten Sultan Khan bei dessen Gegenbesuch in Bonn nur wenige Wochen später außer Acht gelassen.

Insgesamt betrachtet traten Bundesrepublik und DDR nach Abschluss des indisch-sowjetischen Freundschaftsvertrages in Südasien auf der Stelle. Indien hielt weiter zur Bundesrepublik Deutschland und würde die DDR vorerst nicht anerkennen. Die Annäherungsversuche der provisorischen Regierung von Bangladesch wiederum waren für die DDR das Risiko noch nicht wert, Pakistan vor den Kopf zu stoßen. Zudem war Mujibur Rahmans Awami League unter ideologischen Gesichtspunkten kaum ein Wunschpartner der DDR. Um der indischen Regierung zu schmeicheln, bekannte die DDR-Führung sich wenige Tage vor Kriegsausbruch dennoch eindeutig zum indischen Standpunkt. Den Weg zur möglichen Aufnahme von offiziellen Gesprächen mit der pakistanischen Regierung hatte die DDR sich durch diesen Vorstoß allerdings bis auf Weiteres verbaut. 
